\title{
Comparative transcriptome analysis reveals the mechanism of cross-protection against Verticillium wilt conferred on sunflower by hypovirulent strain Gibellulopsis nigrescens $\mathrm{Vn}-1$
}

\section{Xin Zhao}

Inner Mongolia Agricultural University

\section{Bao-Zhu Dong}

Inner Mongolia Agricultural University

Ying-Jie Zhao

Inner Mongolia Agricultural University

Dong Wang

Inner Mongolia Agricultural University

Lan Jing

Inner Mongolia Agricultural University

Hong-You Zhou ( $\sim$ hongyouzhou2002@aliyun.com )

Inner Mongolia Agricultural University

\section{Research Article}

Keywords: Comparative transcriptome, resistance genes, hypovirulent strain, cross-protection, Verticillium wilt

Posted Date: December 15th, 2020

DOI: https://doi.org/10.21203/rs.3.rs-121872/v1

License: (c) (i) This work is licensed under a Creative Commons Attribution 4.0 International License. Read Full License 


\section{Abstract}

Background: Hypovirulent fungal strain Gibellulopsis nigrescens $\mathrm{Vn}-1$ cross-protects sunflower against Verticillium wilt. To discover the mechanism of cross-protection by this hypovirulent strain, we analyzed defense enzyme activities and transcriptomes of root samples infected with virulent and hypovirulent strains.

Results: Defense enzyme activities increased after inoculation, with the highest levels observed $24 \mathrm{~h}$ postinoculation. At the same time, defense enzyme gene expressions were upregulated, and $\mathrm{H}_{2} \mathrm{O}_{2}$ accumulation decreased. A comparative transcriptome analysis revealed that three specific oxidoreductase-related GO terms were significantly enriched in the $\mathrm{Vn}-1$ group compared with the control. In addition, 33 resistance genes and 160 susceptibility genes were predicted. Seven transcription factors (TFs), two phytohormone response factors, two E3 ubiquitin-protein ligases, two CCR4-associated factor 1 proteins, and two predicted leucine repeat rich (LRR) receptors were found to contribute to the conferral of resistance on sunflower.

Conclusions: According to our results, hypovirulent strain G. nigrescens $\mathrm{Vn}-1$ can reduce levels of reactive oxygen species in sunflower induced by infection with virulent strains such as $V$. dahliae V33 by regulating HaCAT expression. Furthermore, multiple resistance-related TFs, phytohormones, and receptors contribute to the formation of specific resistance against virulent strain $V$. dahliae V33.

\section{Introduction}

Many species of Verticillium are globally distributed, soil-borne vascular-wilt fungal pathogens that infect numerous hosts, including sunflower (Helianthus annuus L.) [1], tomato [2], potato [3], lettuce [4-5], cotton [6], and cauliflower, and cause destructive wilt disease [7]. Among members of the genus, $V$. dahliae has the broadest host range, with the ability to infect over 200 plant species [8-9]. Sunflower is a globally important oilseed crop, and sunflower Verticillium wilt caused by $V$. dahliae is one of the most destructive diseases in oilseed crop production [10]. In the absence of hosts, Verticillium species are able to survive in soil for up to 14 years by relying on melanized resting structures [11]. Resting structures of Verticillium, which vary according to species, include microsclerotia, chlamydospores, and melanized resting mycelia [12-15]. In many crops, Verticillium wilt disease is difficult to manage by agricultural and chemical means because these resting structures are present in soil. As a result, the development of resistant cultivars and the enhancement of plant resistance are critical measures to control Verticillium wilt in agricultural production.

Innate and induced resistance (so-called cross-protection) constitute the basal resistance system of plants that protects against biotic and abiotic stresses from the environment [16-18]. When plants recognize pathogen-associated molecular patterns or phytopathogen effectors [19-20], they invoke defense responses, including a hypersensitive response, which is localized, and system acquired resistance (SAR), which is both localized and distal from the primary infection [21-22]. SAR is 
accompanied by the transduction of endogenous signals, including salicylic acid (SA) or jasmonic acid $(\mathrm{JA})$, as well as the expression of pathogenesis-related (PR) proteins and the upregulation of defense enzyme genes [23-25]. Cross-protection is typically used to improve plant resistance against phytopathogens in agricultural production [26], with biotic inducers (including endophytes and hypovirulent strains) and abiotic ones (including physical or chemical agents) commonly used [27].

Hypovirulent or avirulent phytopathogens, which are types of biological inducers, can potentially confer host plant resistance against virulent strains [28]. Cross-protection methods using hypovirulent strains have the potential to control plant disease. For example, plants infected with a hypovirulent virus line are usually resistant to superinfection by other strains of the same virus [29-30]. Cross-protection has been used to control plant virus diseases [31], such as tobacco mosaic virus in tomato [32], zucchini yellow mosaic virus [33], and papaya ringspot virus [34-35]. Cross-protection involving phytopathogenic fungi has also been discovered and used to control plant fungal diseases [36-37]. For example, many destructive phytopathogenic fungi have been effectively controlled by cross-protection using hypovirulent strains, including members of the genera Fusarium, Colletotrichum, Verticillium, and Puccinia [38-39]. Hypovirulent Verticillium strains have been previously isolated from cotton and demonstrated as promising agents for biocontrol of cotton Verticillium wilt [40-41]. Zhao et al. recently isolated a hypovirulent strain of Gibellulopsis nigrescens (strain $\mathrm{Vn}-1$ ) causing minimal wilt in sunflower [42]. This strain was found to confer cross-protection against a virulent strain ( $V$. dahliae V33) on sunflower [42]. Cross-protection functions have also been discovered between different phytopathogens [43]. The exploitation of cross-protection is thus an efficient biocontrol measure for managing plant disease. Moreover, hypovirulent strains confer broad spectrum antifungal activity to protect plants against multiple phytopathogens.

Although cross-protection has long been known, the underlying mechanisms have been poorly understood until recently [44]. With the development of sequencing technology, transcriptome analysis is now the most efficient method for analyzing interactions between hypovirulent strains and host plants [45-46]. Various analytical approaches in combination with transcriptome sequencing are potentially useful for discovering PR genes and ultimately revealing cross-protection mechanisms. These approaches include 1) genome mapping, 2) differentially expressed gene (DEG) analysis, 3) DEG gene ontology (GO) enrichment analysis, and 4) DEG Kyoto Encyclopedia of Genes and Genomes (KEGG) enrichment analysis [47-51].

In this study, we measured expression levels of defense enzyme genes and $\mathrm{H}_{2} \mathrm{O}_{2}$ accumulation and analyzed DEGs responsive to hypovirulent strain $G$. nigrescens $\mathrm{Vn}-1$. Our objectives were as follows: 1 ) detection of $G$. nigrescens cross-protection in sunflower; 2) discovery of DEGs involved in formation of the induced resistance; and 3) elucidation of the mechanism of cross-protection against Verticillium wilt conferred by hypovirulent strain G. nigrescens $\mathrm{Vn}-1$.

\section{Results}




\section{Gibellulopsis nigrescens $\mathrm{Vn}-1$ induced the defense response of sunflower}

We previously identified the hypovirulent strain G. nigrescens $\mathrm{Vn}-1$ as a promising candidate for conferral of cross-protection against sunflower Verticillium wilt [42]. To investigate the mechanism of induced resistance achieved with this hypovirulent strain in the present study, we measured defense enzyme activities in sunflower at different time points after inoculation. We found that the activities of four defense enzymes, namely, peroxidase (POD), superoxide dismutase (SOD), catalase (CAT), and phenylalanine ammonia lyase (PAL), were induced after inoculation. Three of these enzymes (POD, SOD, and CAT) had their highest levels of catalytic activity at $24 \mathrm{~h}$ post-inoculation (hpi). The highest PAL activity occurred at $12 \mathrm{hpi}$, with no significant difference observed between $12 \mathrm{hpi}$ and $24 \mathrm{hpi}$ (Fig. 1A). Defense enzyme activities after inoculation with $G$. nigrescens $\mathrm{Vn}-1$ were higher than those following infection with $V$. dahliae $V 33$, a virulent strain causing more serious symptoms of sunflower Verticillium wilt. A quantity Real Time Polymerase Chain Reaction (qRT-PCR) analysis revealed that the expressions of $\mathrm{HaPOD}, \mathrm{HaSOD}, \mathrm{HaCAT}$, and HaPAL were upregulated at $24 \mathrm{hpi}$ (Fig. 1B). Interestingly, sunflower seedlings inoculated with $G$. nigrescens $V n-1$ or $V$. dahliae $V 33$ exhibited reduced $\mathrm{H}_{2} \mathrm{O}_{2}$ accumulation compared with a control (CK) sample at $24 \mathrm{hpi}$ (Fig. 2). These results indicate that hypovirulent strain $G$. nigrescens $\mathrm{Vn}-1$ was able to induce the defense response of sunflower and that $24 \mathrm{hpi}$ was the critical time point, as expressions of the resistance genes were upregulated. The hypovirulent and virulent strains were both able to clear $\mathrm{H}_{2} \mathrm{O}_{2}$ accumulated in sunflower seedlings during infection.

\section{Overview of Illumina RNA-sequencing data}

To investigate the mechanism of resistance induced by hypovirulent strain $G$. nigrescens $V n-1$, we performed transcriptome sequencing of sunflower root samples inoculated with $G$. nigrescens $V n-1$ or $V$. dahliae V33, as well as a control, at $24 \mathrm{hpi}$. The sequencing results were used for comparative transcriptome analysis. More than 45 million clean reads (>6.5 Gb) were generated per root sample (Table 1). The transcriptome assemblies obtained for the three groups of sunflower roots were pooled and used to assemble complete transcripts based on the sunflower genome. After elimination of incomplete transcripts, $86.17 \%$ to $87.30 \%$ of clean reads were mapped to the reference genome; in total, $83.17 \%$ to $84.34 \%$ of clean reads were uniquely mapped (Fig. 3A, Table S1). More than $95 \%$ of the reads were located within exons (Fig. 3B, Table S1). In this study, a gene was considered to be expressed in a sample if a transcript was detected in the cDNA library for three replicates. Relatively few genes were highly expressed (Fig. 3C). In each group of sunflower root samples, the data from three replicates were highly correlated (Pearson's $r>0.95$ ), thus indicating that the transcriptome profiles were highly reproducible (Fig. 3D).

\section{Functional enrichment analysis of DEGs}

To investigate sunflower genes potentially involved in resistance to Verticillium wilt, we compared the three transcriptomes. We identified a total of 1,790 DEGs between Vn-1 and WT groups, including 404 and 1,386 DEGs with up- and downregulated expressions, respectively. We also identified 3,469 DEGs 
between V33 and WT groups, including 1,204 and 2,445 DEGs with up- and downregulated expressions, respectively. Moreover, 744 DEGs were discovered between Vn-1 and V33 groups, of which 501 and 243 were up- and downregulated, respectively (Fig. 4A). Many more genes were differentially expressed between V33 and CK groups than between Vn-1 and CK groups. Venn diagram analysis of DEGs revealed that 1,167 DEGs were included in either Vn-1 vs. CK or V33 vs. CK comparisons, whereas only 58 DEGs were included in all three comparison groups (Vn-1 vs. CK, V33 vs. CK, and Vn-1 vs. V33) (Fig 4B).

\section{Validation of DEGs}

To confirm the reliability of the DEGs identified in the comparative transcriptome analysis, we randomly selected 16 DEGs that were differentially expressed among the three comparison groups for qRT-PCR verification. The qRT-PCR results for most DEGs were highly correlated with the RNA-seq data (Pearson's $r$ ${ }^{3}$ 0.80); the exception was HannXRQ_Chr01g0021411 (Pearson's $\left.r=0.79\right)$ (Fig. 5). This strong correlation indicates that the RNA-seq data were valid and reliable.

\section{Gene Ontology (GO) enrichment analysis of DEGs}

To functionally characterize DEGs, we performed a GO enrichment analysis. The number of significantly enriched $\mathrm{GO}$ terms in the $\mathrm{V} 33$ vs. CK comparison group was much higher than in $\mathrm{Vn}-1$ vs. CK and Vn-1 vs. V33 comparison groups. The nine most significantly enriched GO terms in the Vn-1 vs. CK group were as follows: oxidation-reduction process, response to oxidative stress, single-organism metabolic process, lipid metabolic process, siroheme biosynthetic process, siroheme metabolic process, heme biosynthetic process, cellular glucan metabolic process, and glucan metabolic process (Fig. 6A, Table S2). Oxidationreduction, lipid metabolic, carbohydrate metabolic, and signaling processes were the major subcategories of processes within the biological process category. These four subcategories were also enriched in the V33 vs. CK comparison group (Fig. 6B, Table S2). In both Vn-1 vs. CK and V33 vs. CK comparison groups, the most abundant $\mathrm{GO}$ term in the cellular component category was extracellular region. In the molecular function category, oxidoreductase activity was the most significantly enriched subcategory in all three comparison groups (Fig. 6, Table S2). According to this GO enrichment analysis of DEGs, enriched resistance-related GO terms in Vn-1 vs. CK and V33 vs. CK comparison groups were highly similar, which suggests that both hypovirulent and virulent strains could induce defense responses at early stage of infection.

To identify GO terms associated with Verticillium wilt resistance in sunflower, we compared the terms significantly enriched in Vn-1 vs. CK and V33 vs. CK groups. We found 34 enriched GO terms common to both groups. In addition, 23 and 57 significantly enriched terms were unique to Vn-1 vs. CK and V33 vs. CK groups, respectively (Fig. 7). We speculated that these GO terms were related to resistance and susceptibility to Verticillium wilt.

\section{KEGG pathway enrichment analysis of DEGs}


DEG functions were also examined by KEGG pathway enrichment analysis. DEGs identified in the three comparison groups were found to be related to $17 \mathrm{KEGG}$ pathway (Table S3). According to the results of the analysis, infection with either hypovirulent or virulent strains influenced the expressions of genes related to the following pathways: biosynthesis of secondary metabolites, biosynthesis of unsaturated fatty acids, fatty acid elongation, fatty acid metabolism, phenylalanine metabolism, phenylpropanoid biosynthesis, and plant-pathogen interaction. Majority of DEGs contributing to plant-pathogen interaction pathway, were down-regulated during the Verticillium species interacting with sunflower (Fig S1). Infection with hypovirulent strain Vn-1 additionally affected the expressions of genes in alanine, aspartate, and glutamate metabolism; cutin, suberin and wax biosynthesis; and ribosome pathways (Table S3, Fig. S2), whereas virulent strain V33 was also able to trigger gene expressions in carbon fixation in photosynthetic organisms, carbon metabolism, citrate cycle (TCA cycle), fatty acid degradation, glycolysis/gluconeogenesis, pentose phosphate, and proteasome pathways (Table S3, Fig. S3). Moreover, only three KEGG pathways were significantly enriched in DEGs in the Vn-1 vs. V33 comparison: biosynthesis of unsaturated acids, fatty acid metabolism, and plant-pathogen interaction (Table S3). These results suggest that genes in many KEGG pathways, including alanine, aspartate, and glutamate metabolism; cutin, suberin and wax biosynthesis; and ribosome pathways, were involved in the specific resistance induced by the hypovirulent strain.

\section{Prediction of genes related to Verticillium wilt resistance in sunflower}

To detect Verticillium wilt resistance genes in sunflower, we analyzed the DEGs uncovered by comparative transcriptome methods. DEGs upregulated in Vn-1 vs. CK and Vn-1 vs. V33 comparison groups but downregulated or not significantly changed in V33 vs. CK were predicted to be resistance genes. As a result, 33 genes were predicted to participate in resistance against $V$. dahliae (Fig. 8). As detailed in Table S4, these predicted resistance genes encode seven transcription factors, two phytohormone response factors, two E3 ubiquitin-protein ligases, and two CCR4-associated factor 1 proteins. In addition, two proteins (with conserved leucine-rich repeat (LRR) domains) encoded by HannXRQ_Chr01g0025331 and HannXRQ_Chr14g0444761 were predicted as resistance-related receptors (Fig. S4).

In contrast to resistance genes, genes related to susceptibility were expected to be upregulated in V33 vs. $\mathrm{CK}$ and $\mathrm{V} 33$ vs. Vn-1 comparison groups and downregulated or unchanged in the $\mathrm{Vn}-1 \mathrm{vs.} \mathrm{CK}$ group. As a result, 160 genes were predicted to be related to susceptibility to Verticillium wilt in sunflower (Fig. 8, Table S5). Further research to verify these potential resistance and susceptibility genes is required.

\section{Discussion}

In a previous study, we found that a hypovirulent Verticillium strain provided cross-protection against virulent Verticillium strains in sunflower. In the present study, we monitored the defense response of sunflower at different time points after inoculation with hypovirulent Verticillium strain $\mathrm{Vn}-1$ and performed a transcriptome analysis of samples at $24 \mathrm{hpi}$. According to our results, $24 \mathrm{hpi}$ was the critical time point for inducing the defense response of sunflower. We also analyzed transcriptome data obtained 
at $24 \mathrm{hpi}$. The strong correlation among data sets from the three biological replicates of each sample are indicative of the high reproducibility and reliability of the transcriptome profiling performed in this study, while the correlation between qRT-PCR and RNA-seq results suggests that the DEG analysis was accurate. As revealed by the transcriptome analysis, three specific oxidoreductase-related $\mathrm{GO}$ terms were significantly enriched in the $\mathrm{Vn}-1$ group compared with the control. Many specific genes were only significantly differentially expressed in the $\mathrm{Vn}-1 \mathrm{vs}$. CK comparison group. This result suggests that hypovirulent strain $\mathrm{Vn}-1$ induces sunflower specific resistance to the virulent strain by regulating expression levels of those DEGs.

In this study, we discovered that the 24-hpi time point was the critical stage of infection, with the highest activities of defense enzymes. During this time, HaPAL, HaPOD, HaCAT, and HaSOD were also upregulated. These results suggest that $V$. dahliae $V n-1$ and $G$. nigrescens induced the defense response of sunflower at $24 \mathrm{hpi}$. At this time point, a small number of germinated conidia attach to the root tip surface, and germ tube starts to penetrate into the adjacent epidermal root [52-53]. These results suggest that a small number of conidia of Verticillium species can induce a defense response in sunflower by secreting pathogenetic factors.

Reactive oxygen species (ROS) are important biotic signaling molecules involved in multiple physiological processes, such as seed germination, biotic or abiotic stress response, root development, and stomatal closure [54-59]. In addition, ROS burst, especially that of $\mathrm{H}_{2} \mathrm{O}_{2}$, can regulate plant innate immunity and contribute to hypersensitive response (HR) [60]. Under normal conditions, ROS are rapidly metabolized by antioxidant enzymes or compounds such as vitamins and glutathione [61-64]. When plants are infected by phytopathogens, however, excessive ROS are generated, which leads to resistance formation [65]. Either directly or indirectly, phytopathogens help eliminate excessive ROS by secreting effectors for further host infection [66]. In this study, V. dahliae V33 and G. nigrescens were able to remove redundant $\mathrm{H}_{2} \mathrm{O}_{2}$ in sunflower seedlings by regulating the activities of $\mathrm{HaCAT}$ and $\mathrm{HaPOD}$. These findings suggest that $V$. dahliae $V 33$ and $G$. nigrescens secrete effectors to reduce $\mathrm{H}_{2} \mathrm{O}_{2}$ levels to facilitate infection of sunflower.

According to our qRT-PCR analysis, HaCAT and HaPOD expression levels in samples inoculated with $G$. nigrescens were significantly higher than those in seedlings infected with $V$. dahliae. Three oxidoreductase-related GO terms (G0:0016651, G0:0016705, and G0:0016627) were only enriched in the $\mathrm{Vn}-1$ vs. CK comparison group. These results suggest that oxidoreductases regulate the concentration of ROS and the specific resistance of sunflower to V. dahliae V33 when induced by G. nigrescens Vn- 1 .

Through comparative transcriptome analysis, we discovered 33 resistance genes specifically upregulated in the $\mathrm{Vn}-1$ vs. CK comparison group. Seven (32.32\%) of these resistance genes encode members of WRKY, MYB, and Zinc-finger CCCH TF families. Previous studies of durum wheat, Arabidopsis, rice, and cotton have revealed the involvement of the Zinc-finger $\mathrm{CCCH}$ protein group in biotic and abiotic stress responses [67-69]. In addition, WRKY-type TFs confer differential tolerance to biotic and abiotic stresses in many plant species [70-73]. Further research has revealed that AtWRKY70 regulates SA- and JA- 
dependent defense signal pathways [74] by regulating the PR gene AtNPR1 [75], with similar results reported in rice [76]. In addition, MYB-type TFs are involved in many resistance process in hosts during phytopathogen infection, such as promoting cuticular wax and glucosinolate biosynthesis [77-78], enhancing the expression of defense-related genes [79], and even triggering programmed plant cell death [80]. These results indicate that hypovirulent strain G. nigrescens promotes the specific resistance of sunflower to $V$. dahliae via many types of TF genes. The functions of the resistance genes predicted in this study need further functional verification.

Finally, two predicted resistance-related proteins (HannXRQ_Chr01g0025331 and HannXRQ_Chr14g0444761) contain LRR domains and may serve as receptors during pathogenesis. Our analysis of conserved domains also revealed that HannXRQ_Chr01g0025331 additionally contains Rx and NB-ARC domains, thus suggesting that this protein is a CC-NB-LRR receptor. Previous studies have indicated that the Rx (CC-NB-LRR) protein confers resistance to Potato Virus X (PVX), with the PVX coat protein acting as the AVR determinant [81-82]. Furthermore, $P b 1$ encodes a CC-NB-LRR protein and confers durable, broad-spectrum resistance to Magnaporthe oryzae in rice [83]. Taking into consideration the gene-for-gene principle, we speculate that HannXRQ_Chr01g0025331 acts as a resistance gene in rice to confer specific resistance against virulent strain $V$. dahliae. In addition, an avirulent gene should be present in hypovirulent strain $\mathrm{Vn}-1$ genome but not in virulent strain $V$. dahliae V33.

In this study, we predicted 33 resistance and 160 susceptibility genes. The much larger proportion of susceptibility genes explains why sunflower cultivar LD5009 is susceptible to Verticillium wilt. This conclusion is consistent with the results of a resistance gene identification study performed by Cao et al [84]. Further functional verification of the resistance and susceptibility genes predicted in this study is needed.

\section{Methods}

\section{Strain culture conditions}

Virulent ( $V$. dahliae V33) and hypovirulent (G. nigrescens $\mathrm{Vn}-1)$ strains were both isolated from sunflower and grown on potato dextrose agar at $26^{\circ} \mathrm{C}$ in darkness.

\section{Sunflower seedling cultivation and inoculation}

The susceptible sunflower variety LD5009 (Beijing Kafry Technology Co., Beijing, China) was used for inoculation. Three sunflower seeds were sown per pot in plastic pots (height $\times$ diameter, $10 \mathrm{~cm} \times 12 \mathrm{~cm}$ ). The pots were maintained under 16-h light/8-h dark conditions for 15 days. The relative humidity and temperature (day/night) was kept at $60 \% / 65 \%$ and $28^{\circ} \mathrm{C} / 26^{\circ} \mathrm{C}$, respectively.

To prepare inoculum, five mycelial plugs ( $5 \mathrm{~mm}$ in diameter) of virulent and hypovirulent strains were excised from 15-day-old colonies, placed in conical flasks with $200 \mathrm{~mL}$ Czapek liquid medium, and cultured in a shaking incubator at $100 \mathrm{rpm}$ for 10 days at $26^{\circ} \mathrm{C}$ [40]. Conidia were then collected from the 
medium by centrifugation for $20 \mathrm{~min}$ at $5,000 \mathrm{~g}$ and diluted with sterilized distilled water to a concentration of approximately $1.0 \times 10^{7}$ conidia $\mathrm{mL}^{-1}$. Seedling inoculation was accomplished by incubating the plastic pots on plates containing $50 \mathrm{~mL}$ of conidial suspension for 40 min until the suspension was totally absorbed by the seedling substrate (2:1 [v/v] vermiculite:sand). Each replicate consisted of 10 pots, with three replicates used for each strain. Sterilized water distilled water was used as a control.

\section{Analysis of defense enzyme activities and detection of $\mathrm{H}_{2} \mathrm{O}_{2}$ deposition}

To analyze whether the hypovirulent strain could induce the defense response of sunflower, $\mathrm{H}_{2} \mathrm{O}_{2}$ staining was performed at $24 \mathrm{hpi}$, and defense enzyme activities were monitored at 12, 24, 48, 72, and $96 \mathrm{hpi}$ using an enzyme activity detection kit (Grace Biotechnology, Suzhou, China) according to the manufacturer's instructions.

\section{RNA extraction and qRT-PCR}

To analyze the expressions of defense enzyme genes and validate the RNA-seq results, we collected and rapidly froze root samples in liquid nitrogen at $24 \mathrm{hpi}$. Total RNA was extracted from the samples using a MiniBEST Plant Extraction kit (Takara, Dalian, China) and reverse transcribed using oligo (dT)18 primer and M-MLV reverse transcriptase (Takara). qRT-PCR amplifications were performed on a Roche LightCycler 96 system (Roche, USA). The $18 \mathrm{~S}$ gene was used as an internal control. Relative expression levels were calculated by the $2^{-\Delta \Delta C t}$ method [85]. Three replicates were performed per biological sample. Primer pairs used in this study are listed in Table S6.

\section{Library preparation for transcriptome sequencing}

To analyze sunflower DEGs induced by hypovirulent strain G. nigrescens $\mathrm{Vn}-1$, sterilized water and the virulent strain $V$. dahliae V33 were used as controls. Total RNA was extracted from sunflower samples at $24 \mathrm{hpi}$. A cDNA library was prepared after purification, terminal repair, A-tailing, sequencing adapter ligation, size selection, and PCR enrichment using a NEBNext Ultra RNA Library Prep kit. Libraries were sequenced on an Illumina HiSeq X Ten RNA-seq platform (Illumina, San Diego, CA, USA).

\section{Data analysis}

The sequencing raw data were recorded in a FASTQ file containing sequence information (reads) and corresponding sequencing quality details. Each sample was treated as an independent biological replicate. After removal of adapter- and poly-N-containing reads and reads of low quality from the raw data, Q20 and Q30 values, GC contents, and sequence duplication levels of the clean data were calculated. Transcriptome assembly was accomplished based on the method described by Grabherr et al [86].

All remaining clean reads were mapped to the $H$. annuus reference genome (accession PRJNA396063 in the NCBI/Resource/Genome database) using TopHat2 software [87]. Sequencing depth and gene 
fragment lengths were examined based on the expected number of fragments per kilobase (FPKM) [88], with an FPKM value of 1 set as the threshold for determining whether a gene was expressed [89]. A violin plot of FPKM distributions was generated to compare gene expression levels under different conditions. The final FPKM for each strain was the mean value of three biological replicates.

\section{Differential expression analysis}

Differential expression of genes between groups was analyzed using DESeq (1.10.1), an R package that provides statistical routines for determining differential expression in digital gene expression data using a model based on the negative binomial distribution. The resulting $P$-values were adjusted using Benjamini and Hochberg's approach for controlling the false discovery rate. Genes with an adjusted $P$-value $<0.05$ according to DESeq were considered to be differentially expressed.

\section{GO and KEGG enrichment analyses}

DEGs were functionally annotated by GO and KEGG pathway enrichment analyses. The GO enrichment analysis was carried out using the GOseq R package based on the Wallenius non-central hypergeometric distribution [90]. KOBAS software was used to test the statistical enrichment of DEGs in KEGG pathways [91].

\section{Statistical procedures}

Data were analyzed using SPSS software, and statistical significance was assessed by Student's $t$-test.

\section{Abbreviations}

G. nigrescens: Gibellulopsis nigrescens; V. dahliae: Verticillium dahliae, ROS: Reactive oxygen species; FPKM: Fragments per kilobase transcript per million mapped reads; GO: Gene Ontology, KEGG: Kyoto encyclopedia of genes and genomes; TF: Transcriptional factor, LRR: Leucine rich repeat; PAMPs: Pathogen associated molecular pattern; HR: Hypersensitive response; SAR: System acquire resistance; PR: Pathogenesis-related, DEGs: Different expression genes, CAT: Catalase; PAL: Phenylalaninammonialyase, POD: Peroxidase, SOD: Superoxide dismutase.

\section{Declarations}

\section{Acknowledgments}

We thank Liwen Bianji, Edanz Group China (www.liwenbianji.cn/ac), for editing the English text of a draft of this manuscript.

\section{Authors囚contributions}


$X Z$ and BZD conceived the project, designed the experiment and wrote the manuscript. LJ, DW, YJZ and HYZ revised manuscript. All authors reviewed the manuscript.

\section{Competing interests}

The authors declare that they have no competing interests.

\section{Consent for publication}

Not applicable

\section{Authors $\rrbracket$ details}

Department of Phytopathology, College of Horticulture and Plant Protection, Inner Mongolia Agricultural University, Hohhot 010010, Inner Mongolia Autonomous region, China.

\section{Funding}

The research was founded by National Public Welfare (Agricultural) Scientific Research Project (201501109), National Natural Science Foundation of China (31572049). Development and demonstration of efficient bacterial fungicide (2017YFD0201101). Funding body had no role in the design of the study and collection, analysis and interpretation of data and in writing the manuscript.

\section{Availability of data and materials}

The datasets used and analyzed during this study are included in the published article [and its supplementary information files].

\section{Ethics approval and consent to participate}

Not applicable

\section{References}

1. Martín-Sanz A, Rueda S, García-Carneros AB, González-Fernández S, Miranda-Fuentes P, CastueraSantacruz S, et al. Genetics, host range, and molecular and pathogenic characterization of Verticillium dahliae from sunflower reveal two differentiated groups in Europe. Front Plant Sci. 2018; 9:288-288.

2. Pegg GF, Young DH. Purification and characterization of chitinase enzymes from healthy and Verticillium albo-atrum-infected tomato plants, and from albo-atrum. Physiol. Plant Pathol.1982, 21(3):399-398.

3. Rowe RC. Synergistic interactions between Verticillium dahliae and Pratylenchus penetrans in potato early dying disease. Phytopathology. 1985; 75(4):412-418. 
4. Vallad GE, Subbarao KV. Colonization of resistant and susceptible lettuce cultivars by a green fluorescent protein-tagged isolate of Verticillium dahliae. Phytopathology. 2008; 98(8):871-885.

5. Powell M, Gundersen B, Miles C, Coats K, Inglis DA. First report of Verticillium Wilt on lettuce (Lactuca sativa) in Washington caused by Verticillium tricorpus. Plant Dis. 2013; 97(7):996-996.

6. Zaki Al, Keen NT, Erwin DC, Implication of vergosin and hemigossypol in the resistance of cotton to Verticillium albo-atrum. Phytopathology. 1972; 62(12):1402-1402.

7. Klosterman SJ, Hayes RJ. A soilless Verticillium Wilt assay using an early flowering lettuce line. Plant Dis. 2009; 93(7):691-698.

8. Inderbitzin P, Davis RM, Bostock RM, Subbarao KV. The ascomycete Verticillium longisporum is a hybrid and a plant pathogen with an expanded host range. PloS One. 2011; 6:e18260.

9. Inderbitzin P, Subbarao KV. Verticillium systematics and evolution: how confusion impedes Verticillium Wilt management and how to resolve it. Phytopathology. 2014; 104(6):564-574.

10. Zhang J, Zhang Y, Yang J, Kang L, Elorm AM, Zhou H, et al. The a-1,6-mannosyltransferase VdOCH1 plays a major role in microsclerotium formation and virulence in the soil-borne pathogen Verticillium dahliae. Fungal Biol-uk. 2019; 123(7):539-546.

11. Wilhelm S. Longevity of the Verticillium wilt fungus in the laboratory and field. Phytopathology. 1955; 45:180-181.

12. Inderbitzin P, Bostock RM, Davis RM, Usami T, Platt HW, Subbarao KV. Phylogenetics and taxonomy of the fungal vascular wilt pathogen Verticillium, with the descriptions of five new species. PloS One. 2011; 6(12):e28341.

13. Karapapa VK, Bainbridge BW, Heale JB. Morphological and molecular characterization of Verticillium longisporum comb, nov. pathogenic to oilseed rape. Mycol. Res. 1997; 101(11):1281-1294.

14. Klosterman SJ, Atallah ZK, Vallad GE, Subbarao KV. Diversity, pathogenicity, and management of Verticillium Annu Rev Phytopathol. 2009; 47(1):39-62.

15. Zhang W, Ren Y, Zhang H, Si N, Zhu X, Qi F, et al. Genetic variations of prevailing Verticillium dahliae isolates from cotton in China. Can. J. Plant. Pathol. 2019; 101:565-578.

16. Subramaniam G. Induction of systemic resistance in crop plants against plant pathogens by plant growth-promoting actinomycetes. Plant Growth Promoting Actinobacteria. 2016; 193-202.

17. Gray WM. Plant defence: a new weapon in the arsenal. Curr Biol. 2002; 12:352-354.

18. Glazebrook J, TsuyumuS, Leach JE, Shiraishi T, Wolpert T. Use of expression profiling to explore disease resistance signaling in plants. Genomic \& genetic analysis of plant parasitism \& defense Japan-US science seminar on plant-pathogen interactions, Shizuoka, Japan, November. 2005.

19. Jones JDG, Dangl JL. The Plant immune system. Nature. 2006; 444(7117):323-329.

20. Ranf S. Sensing of molecular patterns through cell surface immune receptors. Curr. Opin. Plant Biol. 2017; 38:68-77.

21. Durrant WE, Dong X. Systemic acquired resistance. Annu Rev Phytopathol. 2004; 42(1):185-209. 
22. Heidel AJ, Baldwin IT. Microarray analysis of salicylic acid- and jasmonic acid-signalling in responses of nicotiana attenuata to attack by insects from multiple feeding guilds. Plant Cell \& Environ. 2004; 27(11):1362-1373.

23. Jing X, Audenaert K, Hofte M, Vleesschauwer DD. Abscisic acid promotes susceptibility to the rice leaf blight pathogen Xanthomonas oryzae pv. oryzae by suppressing salicylic acid-mediated defenses. PLoS One. 2013; 8(6):e67413.

24. Kunkel BN, Brooks DM. Cross talk between signaling pathways in pathogen defense. Curr. Opin. Plant Biol. 2002; 5(4):325-331.

25. Farmer EE, Alméras $\mathrm{E}$, Krishnamurthy $\mathrm{V}$. Jasmonates and related oxylipins in plant responses to pathogenesis and herbivory. Curr. Opin. Plant Biol. 2003; 6(4):372-378.

26. Misaghi IJ, Ayres PG. The physiology of diseased plants effect of disease on the physiology of the growing plant. BioScience. 1982; 32(9).

27. Francia D, Demaria D, Calderini O, Ferraris L, Valentino D, Arcioni S, et al. Wounding induces resistance to pathogens with different lifestyles in tomato: role of ethylene in cross-protection. Plant Cell Environ. 2010; 30(11):1357-1365.

28. Herr LJ. Biological control of Rhizoctonia solani by binucleate Rhizoctonia spp. and hypovirulent solani agents. Crop Prot. 1995; 14(3):179-186.

29. Mckinney HH. Mosaic diseases in the Canary Islands, West Africa and Gibraltar. J Agric Res. 1929; 39(3):577-578.

30. Ziebell H, Carr JP. Cross-protection: a century of mystery. Adv Virus Res. 2010; 76:211-264.

31. Fulton RW. Practices and precautions in the use of cross protection for plant virus disease control. Annu Rev Phytopathol. 1986; 24(1):67-81.

32. Vanderveken J, Coutisse S. Control of tobacco mosaic virus in tomato by cross protection. International Symposium Over Fytofarmacie En Fytiatrie. 1975; 27(2):791-797.

33. Lecoq H, Lemaire, JM, Wipf-Scheibel C. Control of zucchini yellow mosaic virus in squash by cross protection. Plant Dis. 1991; 75(2):208-211.

34. Yeh SD, Gonsalves D, Wang HL, Namba R, Chiu RJ. Control of papaya ringspot virus by cross protection. Plant Dis. 1988; 72(5):375-380.

35. Wang HL, Yeh SD, Chiu RJ, Gonsalves D. Effectiveness of cross-protection by mild mutants of papaya ringspot virus for control of ringspot disease of papaya in Taiwan. Plant Dis. 1987; 71(6):491-497.

36. Ogawa K, Komada H. Biological control of Fusarium Wilt of Sweet Potato with cross-protection by prior inoculation with nonpathogenic Fusarium oxysorum. Jarq-Jpn Agr Res Q, 1985,19.

37. Sutton DC. Systemic cross protection in bean against Colletotrichum lindemuthianum. Australas Plant Path. 1979; 8(1):4-5.

38. Yarwood CE. Cross protection with two rust fungi. Phytopathology. 1956; 46:540-544. 
39. Kochman JK, Brown JF. Studies on the mechanism of cross-protection in Cereal Rusts. Physiol. Plant Pathol. 1975; 6(1):19-27.

40. Zhu HQ, Feng ZL, Li ZF, Shi YL, Zhao YQ, Yang JR. Characterization of two fungal isolates from cotton and evaluation of their potential for biocontrol of Verticillium Wilt of cotton. J Phytopathol. 2013; 161(2):70-77.

41. Vagelas I, Leontopoulos S. Cross-protection of cotton against Verticillium Wilt by Verticillium Emir J Food Agr. 2015; 27(9):687-691.

42. Zhao Y, Wang D, Zhao X, Zhao J, Jing L, Zhou H. Screening of hypovirulent strains and optimization of induction condition for its cross-protection against Verticillium wilt on sunflower. Chinese journal of biological control. 2019; 35(01):112-119. (In Chinese)

43. Chamberlain DW. Heat-induced susceptibility to nonpathogens and cross-protection against Phytophthora megasperma var. sojae in soybean. Phytopathology. 1972; 62(6):645-646.

44. Ziebell H. Mechanism(s) of cross-protection. CAB reviews perspectives in Agriculture Veterinary ence Nutrition and Natural Resources. 2008; 3:13.

45. Zeller G, Henz SR, Widmer CK, Sachsenberg T, Laubinger S, et al. Stress-induced changes in the arabidopsis thaliana transcriptome analyzed using whole-genome tiling arrays. Plant J, 2010; 58(6):1068-1082.

46. Nikiforova V, Freitag J, Kempa S, Hesse H, Bielecka M. Transcriptome analysis of sulfur depletion in arabidopsis thaliana: interlacing of biosynthetic pathways provides response specificity. Plant $\mathrm{J}$. 2010; 33(4):633-50.

47. Boddu J, Svabek C, Ibraheem F, Jones AD, Chopra S. Characterization of a deletion allele of a sorghum Myb gene yellow seed1 showing loss of 3-deoxyflavonoids. Plant Sci. 2005; 169(3):542552.

48. Shankar R, Bhattacharjee A, Jain MT. Transcriptome analysis in different rice cultivars provides novel insights into desiccation and salinity stress responses. Sci. Rep-UK. 2016; 6:23719.

49. Jayaswall K, Mahajan P, Singh G, Parmar R, Seth R, Raina A, et al. Transcriptome analysis reveals candidate genes involved in blister blight defense in tea (Camellia sinensis ( $L$ ) Kuntze).Sci. Rep-UK. 2016; 6:30412.

50. Yang $\mathrm{H}$, Zhao T, Jiang J, Chen X, Zhang H, Liu G, et al. Transcriptome analysis of the sm-mediated hypersensitive response to stemphylium lycopersici in tomato. Front Plant Sci, 2017, 8:1257-1257.

51. Liu Y, Zhang Z, Fu J, Wang G, Wang J, Liu Y. Transcriptome analysis of maize immature embryos reveals the roles of cysteine in improving agrobacterium infection efficiency. Front Plant Sci. 2017; 8:1778.

52. Zhang Y, Zhang J, Gao J, Zhang G, Yu Y, Zhou H, et al. The colonization process of sunflower by a green fluorescent protein-tagged isolate of Verticillium dahliae and its seed transmission. Plant Dis. 2018; 102(9):1772-1778.

53. Zhang WW, Jiang TF, Cui X, Qi FJ, Jian GL. Colonization in cotton plants by a green fluorescent protein labelled strain of Verticillium dahliae. Eur J Plant Pathol. 2012; 135(4):867-876. 
54. Grant JJ, Loake GJ. Role of reactive oxygen intermediates and cognate redox signaling in disease resistance. Plant Physiol. 2000; 124(1):21-29.

55. Schopfer P, Plachy C, Frahry G. Release of reactive oxygen intermediates (superoxide radicals, hydrogen peroxide, and hydroxyl radicals) and peroxidase in germinating radish seeds controlled by light, gibberellin, and abscisic acid. Plant Physiol. 2001; 125(4):1591-602.

56. Ren $D$, Yang $H$, Zhang S. Cell death mediated by MAPK is associated with hydrogen peroxide production in arabidopsis. J. Biol. Chem. 2002; 277(1):559-565.

57. Bindschedler LV, Dewdney J, Blee KA, Stone JM, Asai T, Plotnikov J. et al, Peroxidase-dependent apoplastic oxidative burst in arabidopsis required for pathogen resistance. Plant J. 2006; 47(6):85163.

58. Li SW, Xue LG, Xu SJ, Feng HY, An LZ. Plant growth regulation hydrogen peroxide involvement in formation and development of adventitious roots in cucumber. J. Plant Growth Regul. 2007; 52:173180.

59. Torres MA. ROS in biotic interactions. Physiol. Plant. 2009; 138(4):414-429.

60. Yoshioka H, Asai S, Yoshioka M, Kobayashi M. Molecular mechanisms of generation for nitric oxide and reactive oxygen species, and role of the radical burst in plant immunity. Mol Cells, 2009; 28(4):321-9.

61. Kovtun Y, Chiu WL, Tena G, Sheen J. Functional analysis of oxidative stress-activated mitogenactivated protein kinase cascade in plants. Proc. Natl. Acad. Sci. USA. 2000; 97(6):2940-5

62. Lim YS, Cha MK, Kim HK, Uhm TB, Park JW, Kim K, et al. Removals of hydrogen peroxide and hydroxyl radical by thiol-specific antioxidant protein as a possible role in vivo. Biochem Biophy Res Co. 1993, 192(1):273-280.

63. Scandalios, JG. Molecular biology of the antioxidant defense genes encoding catalases and superoxide dismutases in maize. In: Hatzios KK, editor. Regulation of enzymatic systems detoxifying xenobiotics in plants, Dordrecht: Kluwer Academic Publishers; 1997.

64. Alscher RG. Biosynthesis and antioxidant function of glutathione in plants. Physiol. Plant. 1989; 77(3):457-464.

65. Doke N. Mechanisms of active defence in host plants and race-specific pathogenicity of pathogen in potato late blight. Japanese J. Phytopathol.. 1997; 63(3):130-132.

66. Nováková M, Vladimír S, Trdá L, Krutinová H, Mongin T, Valentová O, et al. Leptosphaeria maculans effector AvrLm4-7 affects salicylic acid (SA) and ethylene (ET) signalling and hydrogen peroxide $\left(\mathrm{H}_{2} \mathrm{O}_{2}\right)$ accumulation in Brassica napus. Mol. Plant Pathol. 2016; 17(6):818-831.

67. Guo YH, Yu YP, Wang D, Wu CA, Yang GD, Huang JG, et al. GhZFPI, a novel CCCH-type zinc finger protein from cotton, enhances salt stress tolerance and fungal disease resistance in transgenic tobacco by interacting with GZIRD2IA and GZIPR5. New Phytol. 2009; 183(1):62-75.

68. De Leonardis AM, Dorso F, Morelli G, Ruberti R, Cattivelli L, Mastrangelo AM. A conserved group of $\mathrm{CCCH}$ zinc finger proteins is involved in response to abiotic stresses in durum wheat and 
Arabidopsis. J. Biotechnol. 2010; 150:491-491.

69. Michael DHH, Kazue IDH, Dulal B. Identification of drought-induced genes in giant leucaena (Leucaena leucocephala glabrata). Trees Struct. Funct. 2018; 32(2):571-585.

70. Ülker B, Somssich IE. WRKY transcription factors: from DNA binding towards biological function. Curr. Opin. Plant Biol. 2004; 7(5):491-498.

71. Zhou QY, Tian AG, Zou HF, Xie ZM, Lei G, Huang J, et al. Soybean WRKY-type transcription factor genes, GmWRKY13, GmWRKY21, and GmWRKY54, confer differential tolerance to abiotic stresses in transgenic Arabidopsis plants. Plant Biotechnol. J. 2008; 6(5):486-503.

72. Zhou L, Wang N, Gong S, Lu R, Li Y, Li X. Overexpression of a cotton (Gossypium hirsutum) WRKY gene, GhWRKY34, in Arabidopsis enhances salt-tolerance of the transgenic plants. Plant Physiol. Bioch. 2015; 96:311-320.

73. Naoki Y, Yuko S, Shigeru T, Tetsuya C, Takafumi S, Kazunori K, et al. WRKY76 is a rice transcriptional repressor playing opposite roles in blast disease resistance and cold stress tolerance. J. Exp. Bot. 2013; (16):5085-5097.

74. Li J, Brader G, Palva ET. The WRKY70 transcription factor: a node of convergence for jasmonatemediated and salicylate-mediated signals in plant defense. Plant Cell. 2004; 16(2):319-331.

75. Yu D, Chen C, Chen Z. Evidence for an important role of WRKY DNA binding proteins in the regulation of NPR1 gene expression. Plant Cell. 2001; 13(7):1527-1540.

76. Liu X, Bai X, Qian Q, Wang X, Chen M, Chu C. OsWRKY03, a rice transcriptional activator that functions in defense signaling pathway upstream of OsNPR1. Cell Res. 2005; 8:593-603.

77. Zhang YL, Zhang CL, Wang GL, Wang YX, Hao YJ. The R2R3 MYB transcription factor MdMYB30 modulates plant resistance against pathogens by regulating cuticular wax biosynthesis. BMC Plant Biol. 2019; 19(1): 362-362.

78. Yatusevich R. Analysis of the MYB28, MYB29 and MYB76 transcription factors involved in the biosynthesis of aliphatic glucosinolates in Arabidopsis thaliana.

79. Liu Z, Luan Y, Li J, Yin Y. Expression of a tomato MYB gene in transgenic tobacco increases resistance to fusarium oxysporum and botrytis cinerea. Eur J Plant Pathol. 2016; 144:607-617.

80. Daniel X, Lacomme C, Jean-Benoît Morel, Roby D. A novel myb oncogene homologue in arabidopsis thaliana related to hypersensitive cell death. The Plant J. 1999; 20(1):57-66.

81. Bendahmane A, Köhn BA, Dedi C, Baulcombe DC. The coat protein of potato virus X is a strainspecific elicitor of Rx1-mediated virus resistance in potato. The Plant J. 1996; 8(6):933-941.

82. Rairdan GJ, Moffett P. Distinct domains in the ARC region of the potato resistance protein Rx mediate LRR binding and inhibition of activation. Plant Cell. 2006; 18(8):2082-2093.

83. Inoue H, Hayashi N, Matsushita A, Liu X, Nakayama A, Sugano S, et al. Blast resistance of CC-NB-LRR protein $\mathrm{Pb} 1$ is mediated by WRKY45 through protein-protein interaction. PNAS. 2013; 110(23):95779582. 
84. Cao, X, Meng, Q, Liu, J, Li, X, Bu, H, Ren, J, et al. The identification of resistance of different sunflower varieties to Verticillium wilt in fields. Crops. 2014; 35(1):67-72. (In Chinese)

85. Livak KJ, Schmittgen TD. Analysis of relative gene expression data using real-time quantitative PCR and the $2^{-\triangle \Delta C T}$ Methods. 2001;25(4):402-408.

86. Garber M, Grabherr MG, Guttman M, Trapnel C. Computational methods for transcriptome annotation and quantification using RNA-seq. Nat Methods. 2011; 8(6):469-77.

87. Kim D, Pertea G, Trapnell C, Pimentel H, Relley R, Salzberg SL. TopHat2: accurate alignment of transcriptomes in the presence of insertions, deletions and gene fusions. Genome Biol. 2013; 14(4):R36.

88. Trapnell C, Williams A, Pertea G, Mortazavi A, Kwan G, Baren MJ, et al. Transcript assembly and quantification by RNA-Seq reveals unannotated transcripts and isoform switching during cell differentiation. Nat. Biotechnol. 2010;28(5):511-515.

89. Anders S, Pyl PT, Huber W. HTSeq-a python framework to work with high-throughput sequencing data. Bioinformatics. 2015; 31(2):166-169.

90. Kim KY, Shin YK, Kang KC, Yoo JS, Jung-Ho K, Kim JH. Proteomic profiling of yeast- and hyphalspecific responses of Candida albicans to the antifungal agent, HWY-289. Proteom Clin Appl. 2009; 3(4):452-461.

91. Mao X, Cai T, Olyarchuk JG, Wei L. Automated genome annotation and pathway identification using the KEGG orthology (KO) as a controlled vocabulary. Bioinformatics. 2005; 21(19):3787-3793.

\section{Tables}

Table 1 Statistical results of transcriptome sequencing for sunflower root samples of three groups

\begin{tabular}{|llllllll|}
\hline $\begin{array}{l}\text { Sample } \\
\text { name }\end{array}$ & $\begin{array}{l}\text { Raw } \\
\text { reads }\end{array}$ & $\begin{array}{l}\text { Clean } \\
\text { reads }\end{array}$ & $\begin{array}{l}\text { Clean base } \\
(\mathrm{G})\end{array}$ & $\begin{array}{l}\text { Error rate } \\
(\%)\end{array}$ & $\begin{array}{l}\text { Q20 } \\
(\%)\end{array}$ & $\begin{array}{l}\text { Q30 } \\
(\%)\end{array}$ & $\begin{array}{l}\text { GC content } \\
(\%)\end{array}$ \\
\hline CK_1 & 61083664 & 58700570 & 8.81 & 0.01 & 97.58 & 93.84 & 44.15 \\
\hline CK_2 & 48462484 & 46612246 & 6.99 & 0.01 & 97.72 & 94.15 & 44.34 \\
\hline CK_3 & 48556692 & 46848766 & 7.03 & 0.01 & 97.64 & 93.96 & 44.26 \\
\hline Vn-1_1 & 47695750 & 46140060 & 6.92 & 0.01 & 97.65 & 94.00 & 44.43 \\
\hline Vn-1_2 & 58714830 & 56815164 & 8.52 & 0.01 & 97.53 & 93.76 & 44.37 \\
\hline Vn-1_3 & 48462128 & 46811500 & 7.02 & 0.01 & 97.62 & 93.96 & 44.33 \\
\hline V33_1 & 50296234 & 48645648 & 7.30 & 0.01 & 97.72 & 94.21 & 44.39 \\
\hline V33_2 & 52306524 & 50546856 & 7.58 & 0.01 & 97.72 & 94.21 & 44.45 \\
\hline V33_3 & 48559622 & 49660786 & 7.04 & 0.01 & 97.86 & 94.47 & 44.38 \\
\hline
\end{tabular}




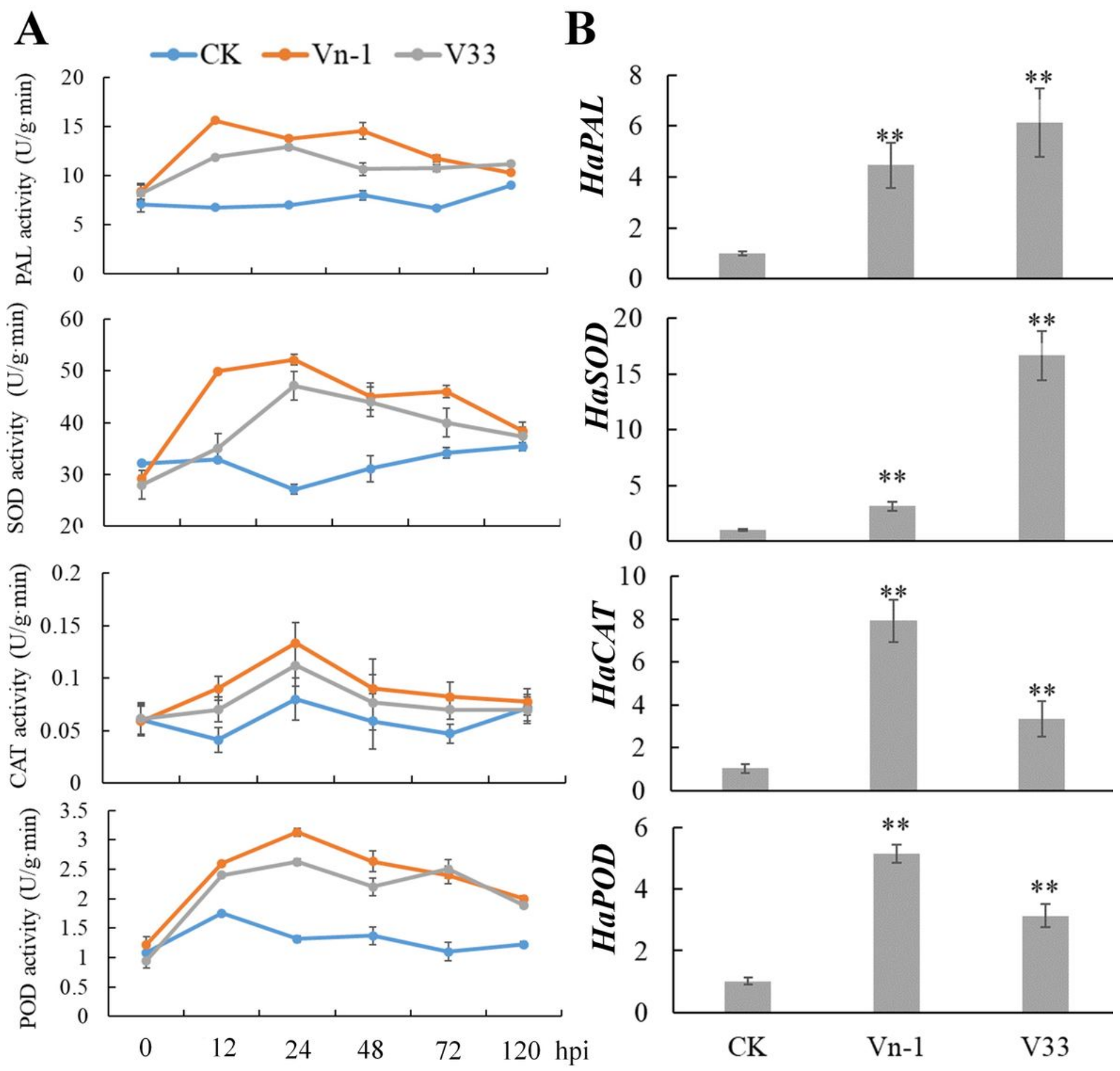

Figure 1

Defense enzyme activities and gene expression levels A. Defense enzyme activities of sunflower at different time points after inoculation with hypovirulent (Vn-1) and virulent (V33) strains of Verticillium. B. Relative expressions of defense enzyme genes $24 \mathrm{~h}$ post-inoculation (hpi). Error bars indicate standard deviation (SD) based on three replicates. Statistical significance between Vn-1/V33 and CK was analyzed using student's t-test, asterisks indicate significant differences ( $\left.{ }^{*}, \mathrm{P}<0.05 ; * \star, \mathrm{P}<0.01\right)$. 
A

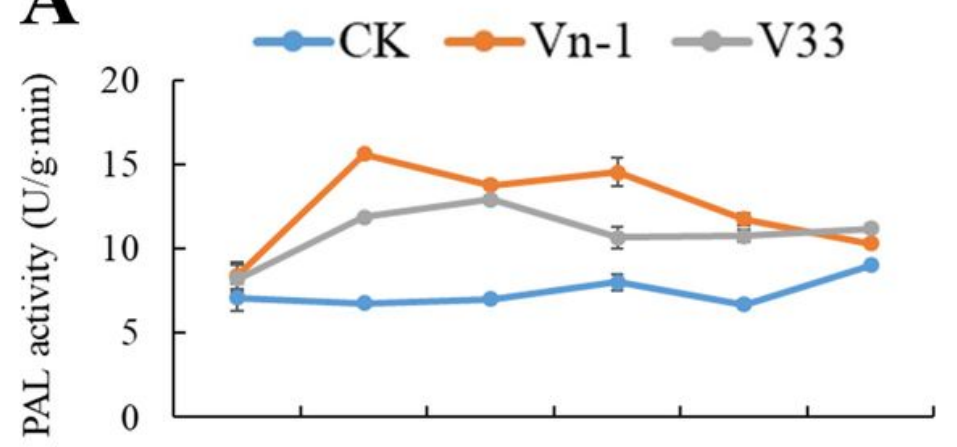

B
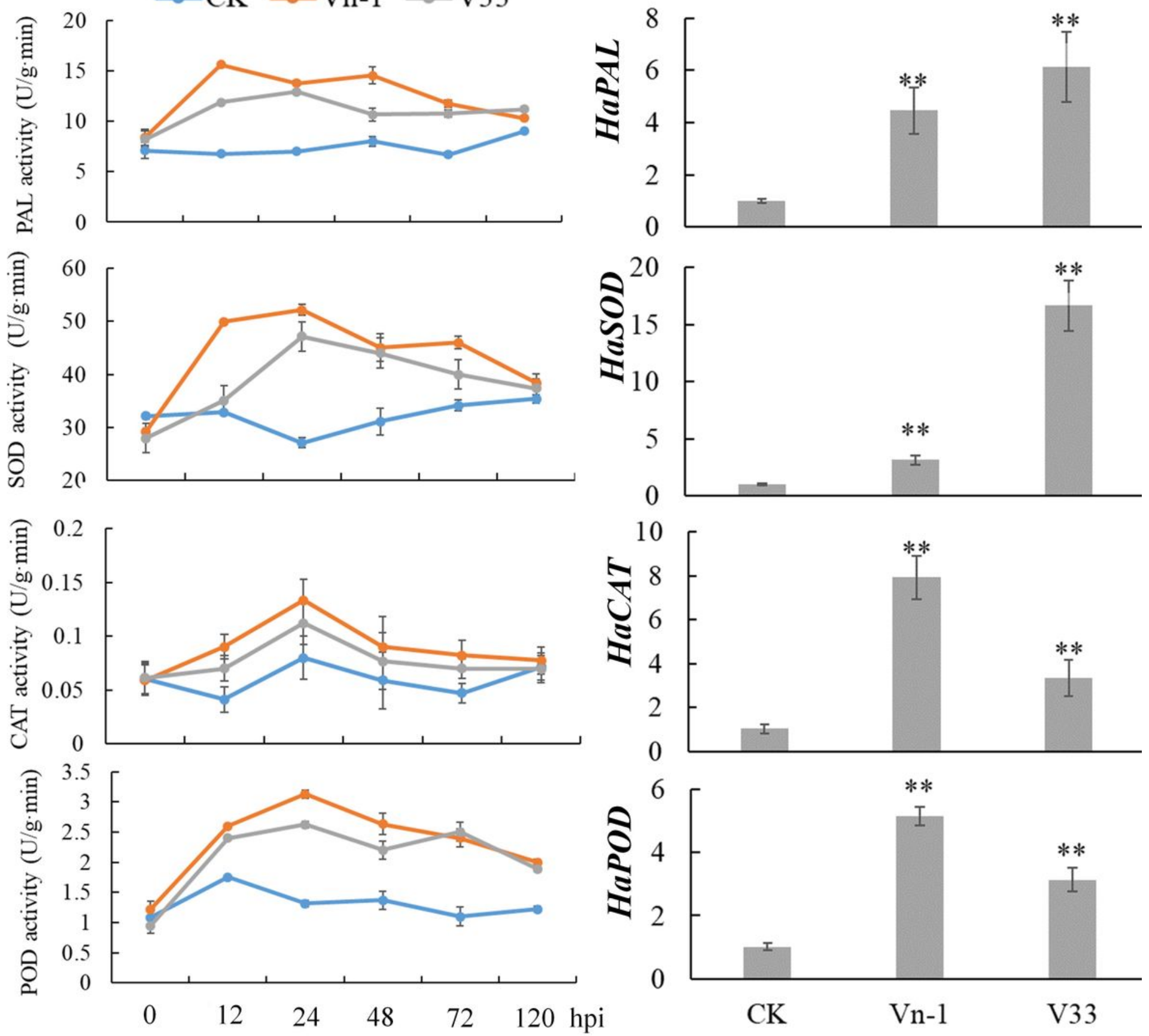

Figure 1

Defense enzyme activities and gene expression levels A. Defense enzyme activities of sunflower at different time points after inoculation with hypovirulent (Vn-1) and virulent (V33) strains of Verticillium. B. Relative expressions of defense enzyme genes $24 \mathrm{~h}$ post-inoculation (hpi). Error bars indicate standard deviation (SD) based on three replicates. Statistical significance between Vn-1/V33 and CK was analyzed using student's t-test, asterisks indicate significant differences ( $\left.{ }^{*}, \mathrm{P}<0.05 ; * \star, \mathrm{P}<0.01\right)$. 


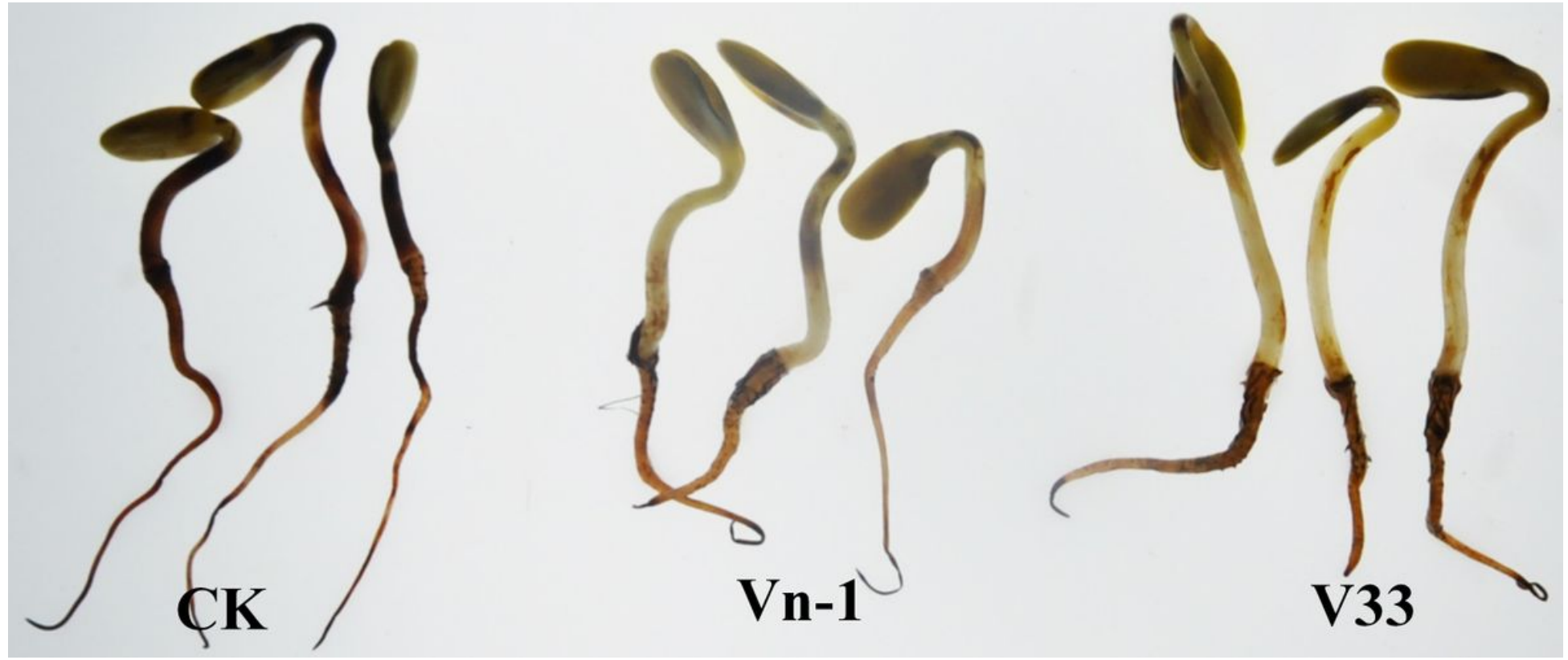

Figure 2

$\mathrm{H} 2 \mathrm{O} 2$ deposition in sunflower seedlings at 24 hour-post inoculation (hpi) Accumulated $\mathrm{H} 2 \mathrm{O} 2$ (brown areas) in 24-hpi sunflower seedlings stained with DAB.
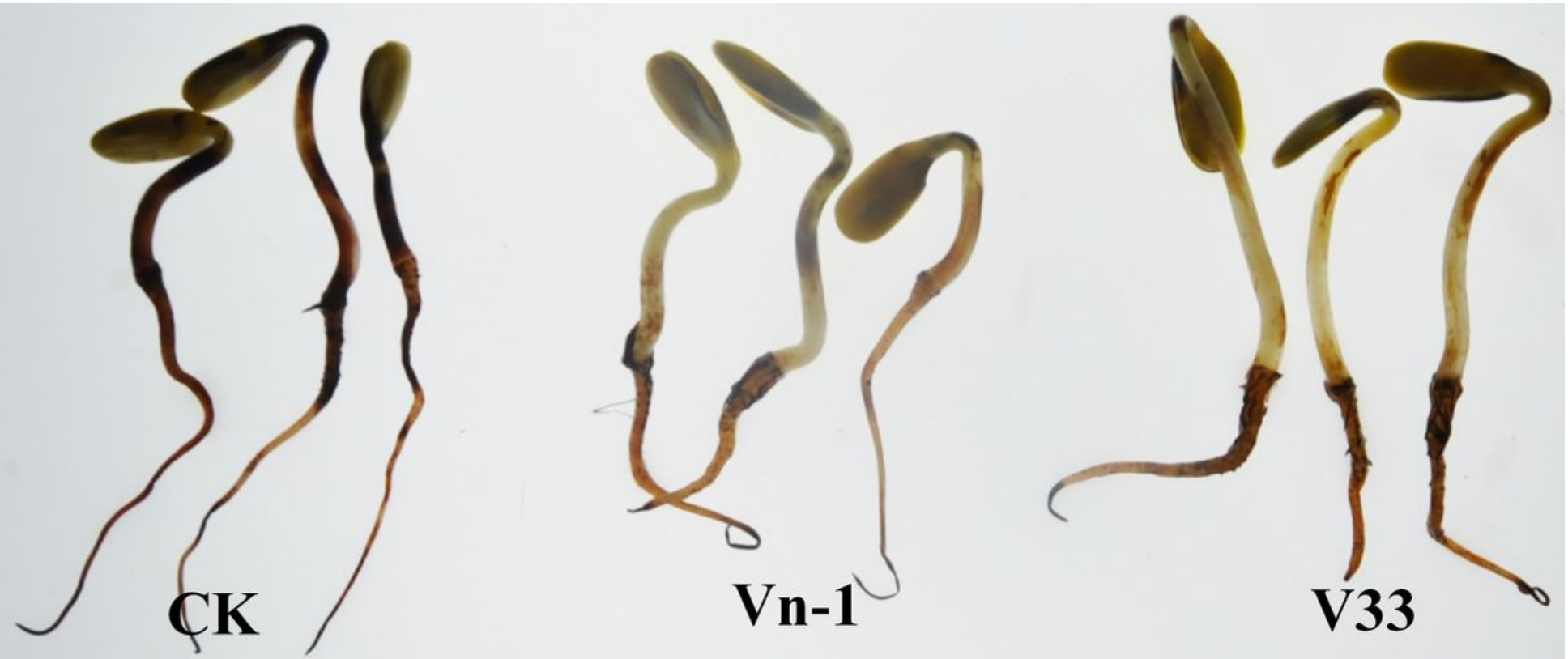

Figure 2

$\mathrm{H} 2 \mathrm{O} 2$ deposition in sunflower seedlings at 24 hour-post inoculation (hpi) Accumulated H2O2 (brown areas) in 24-hpi sunflower seedlings stained with DAB. 


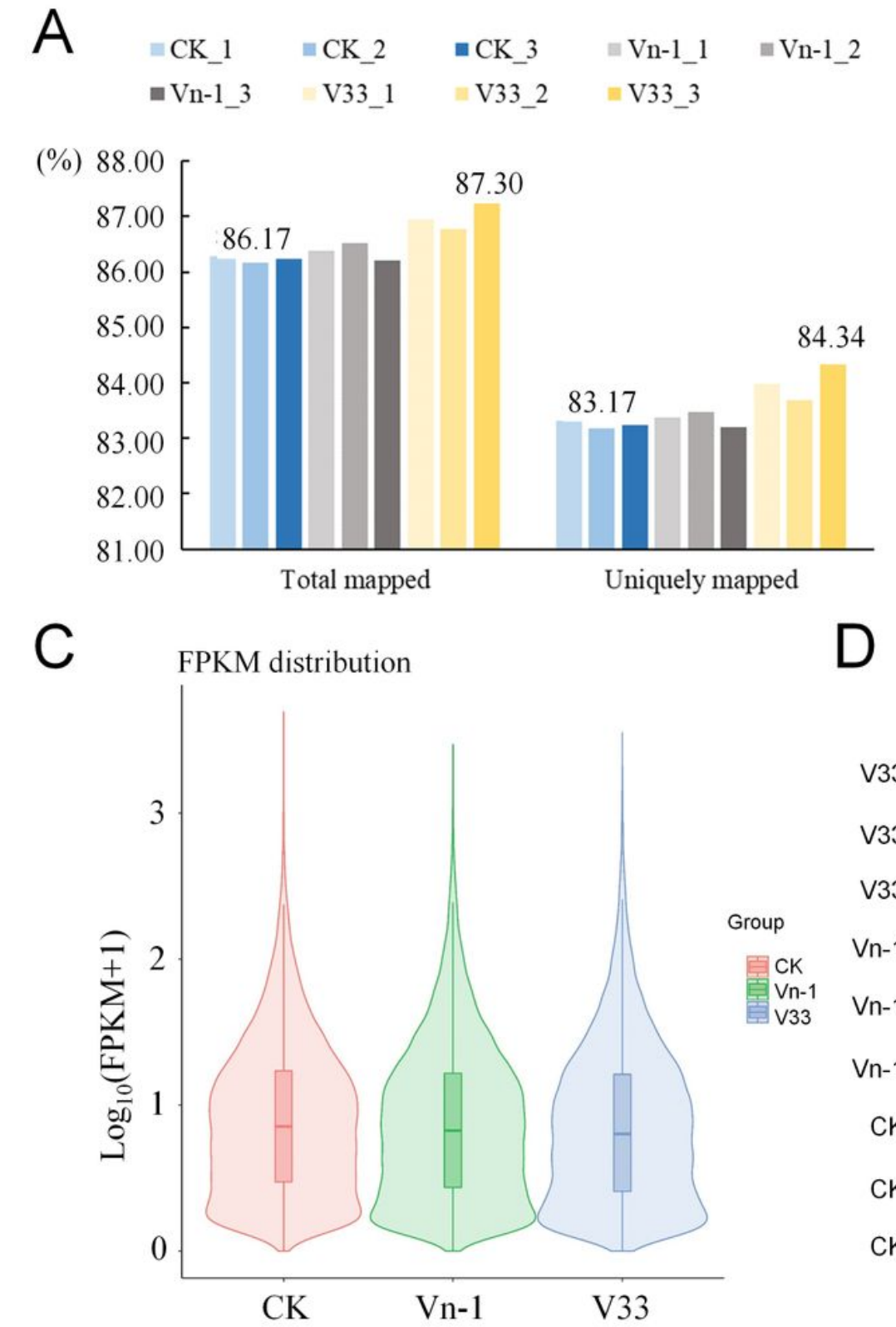

B घ Exon $=$ Intergenic $=$ Intron

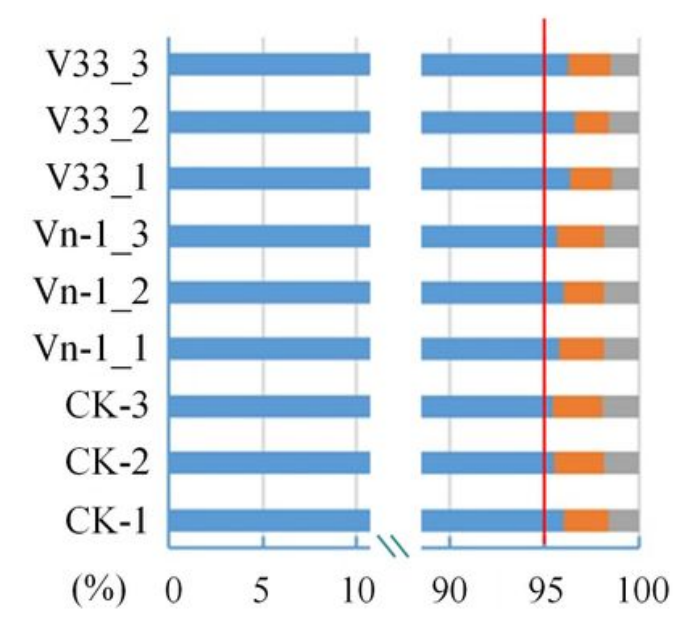

$\mathrm{D}$

Pearson correlation between smples

\section{Figure 3}

Analysis of transcriptome data mapped to the sunflower (Helianthus annuus L.) genome A. Percentage of total clean reads from three treatments (three samples per treatment) mapped to the sunflower genome. B. Percentage of clean reads located in exons, introns, and intergenic regions. C. Violin plots of gene expression levels based on FPKM values in various samples. The $x$-axis indicates sample names, and the $y$-axis represents $\log 10(F P K M+1)$ values. Each violin plot includes five statistical measures (maximum value, upper quartile, median, lower quartile, and minimum value). The width of a violin is proportional to gene density. D. Correlation analysis of RNA-seq data. Heat maps of the correlation between samples are shown. R2, the square of the Pearson's correlation coefficient. 

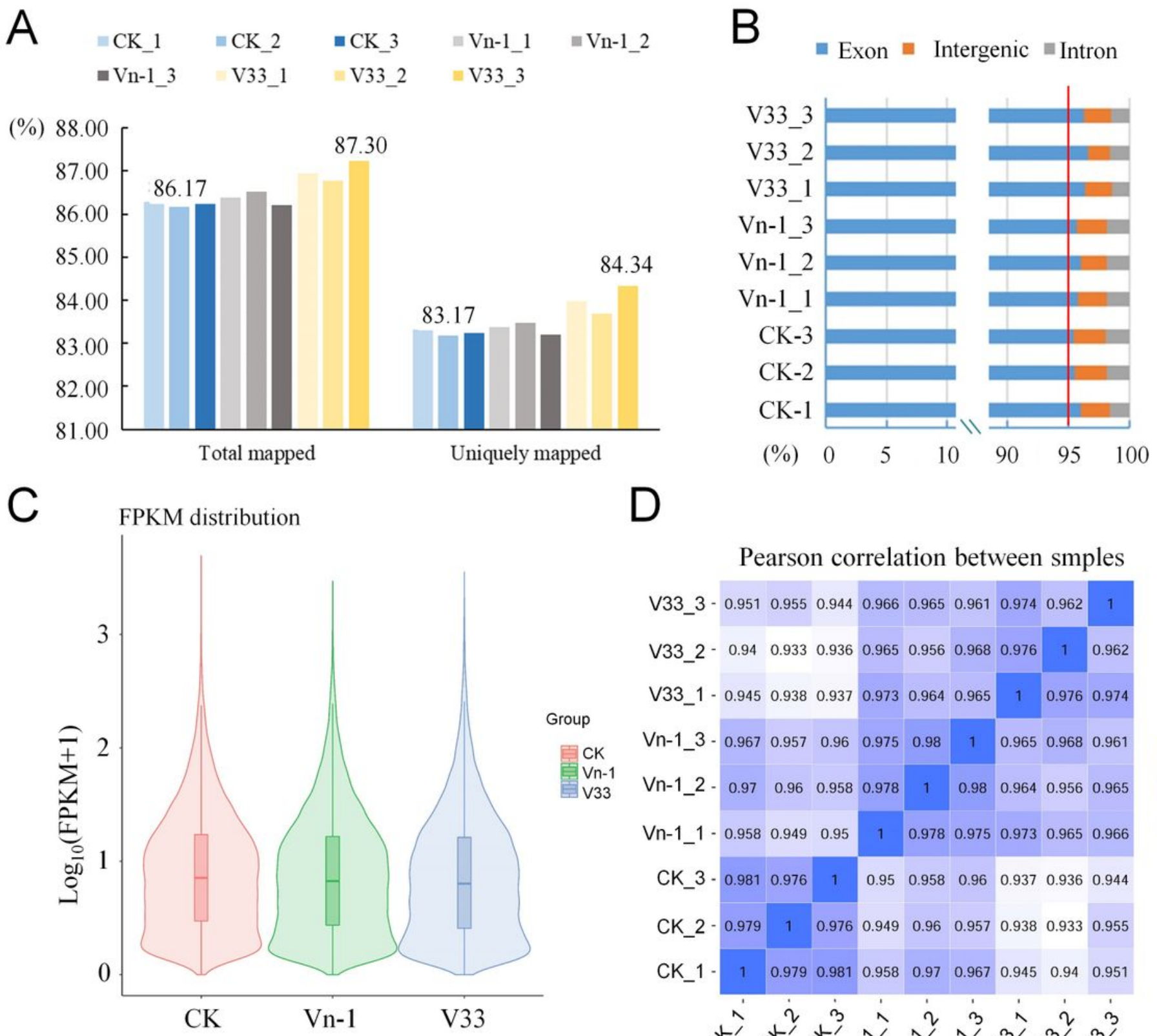

D

Pearson correlation between smples

\section{Figure 3}

Analysis of transcriptome data mapped to the sunflower (Helianthus annuus L.) genome A. Percentage of total clean reads from three treatments (three samples per treatment) mapped to the sunflower genome. B. Percentage of clean reads located in exons, introns, and intergenic regions. C. Violin plots of gene expression levels based on FPKM values in various samples. The $x$-axis indicates sample names, and the $y$-axis represents $\log 10(F P K M+1)$ values. Each violin plot includes five statistical measures (maximum value, upper quartile, median, lower quartile, and minimum value). The width of a violin is proportional to gene density. D. Correlation analysis of RNA-seq data. Heat maps of the correlation between samples are shown. R2, the square of the Pearson's correlation coefficient. 


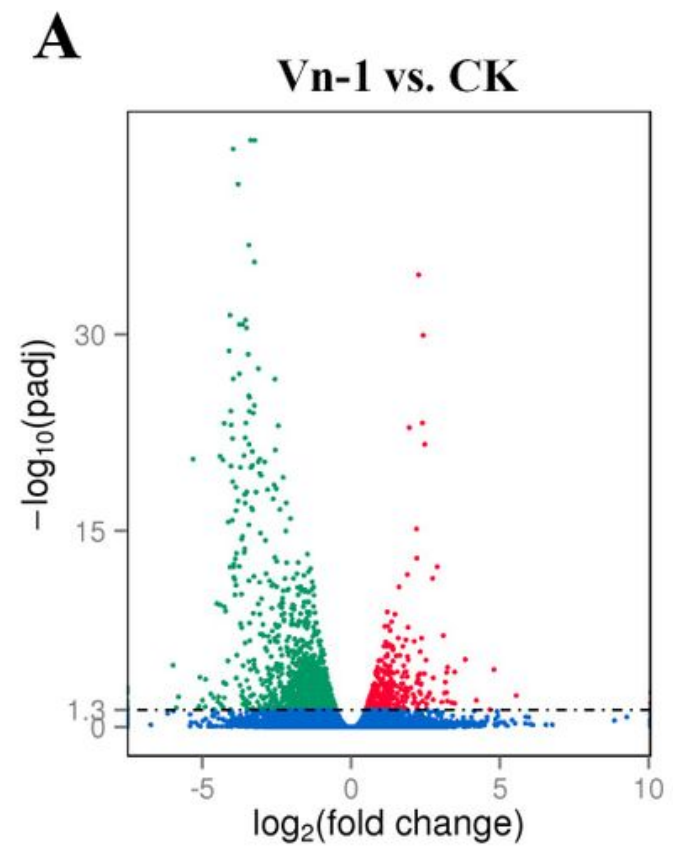

Differential Expressed Genes ( 1790 )

- up regulated: 404

down regulated: 1386

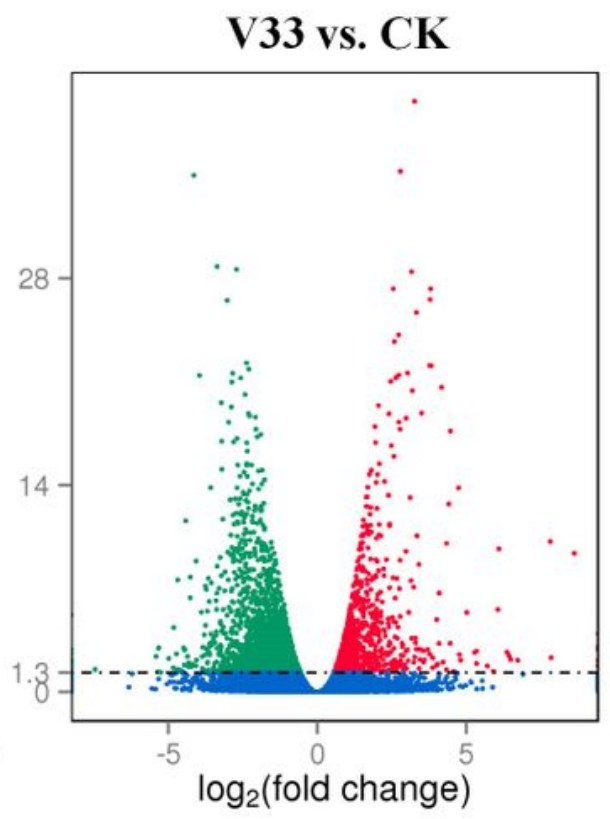

Differential Expressed Genes ( 3649 )

up regulated: 1204

down regulated: 2445

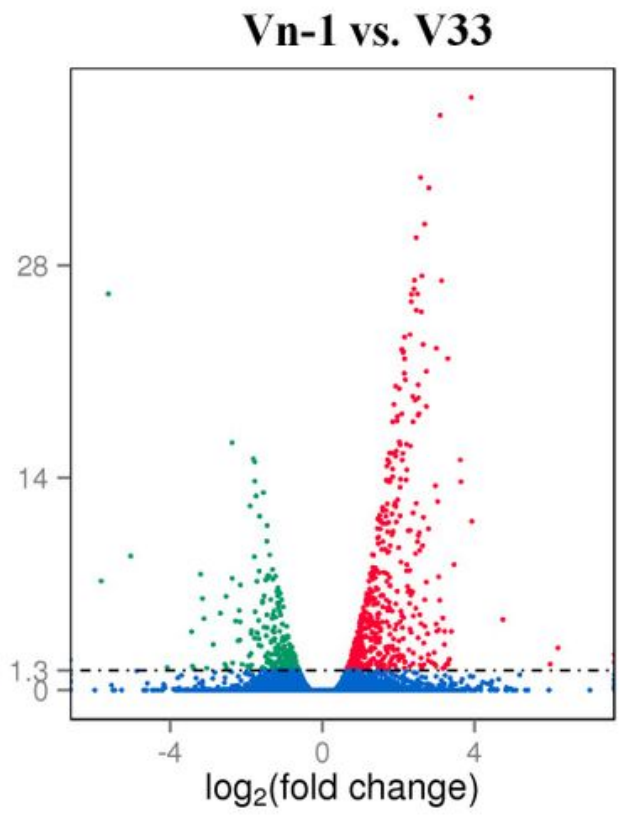

Ditterential Expressed Genes ( 744 )

- up regulated: 501

down regulated: 243

B

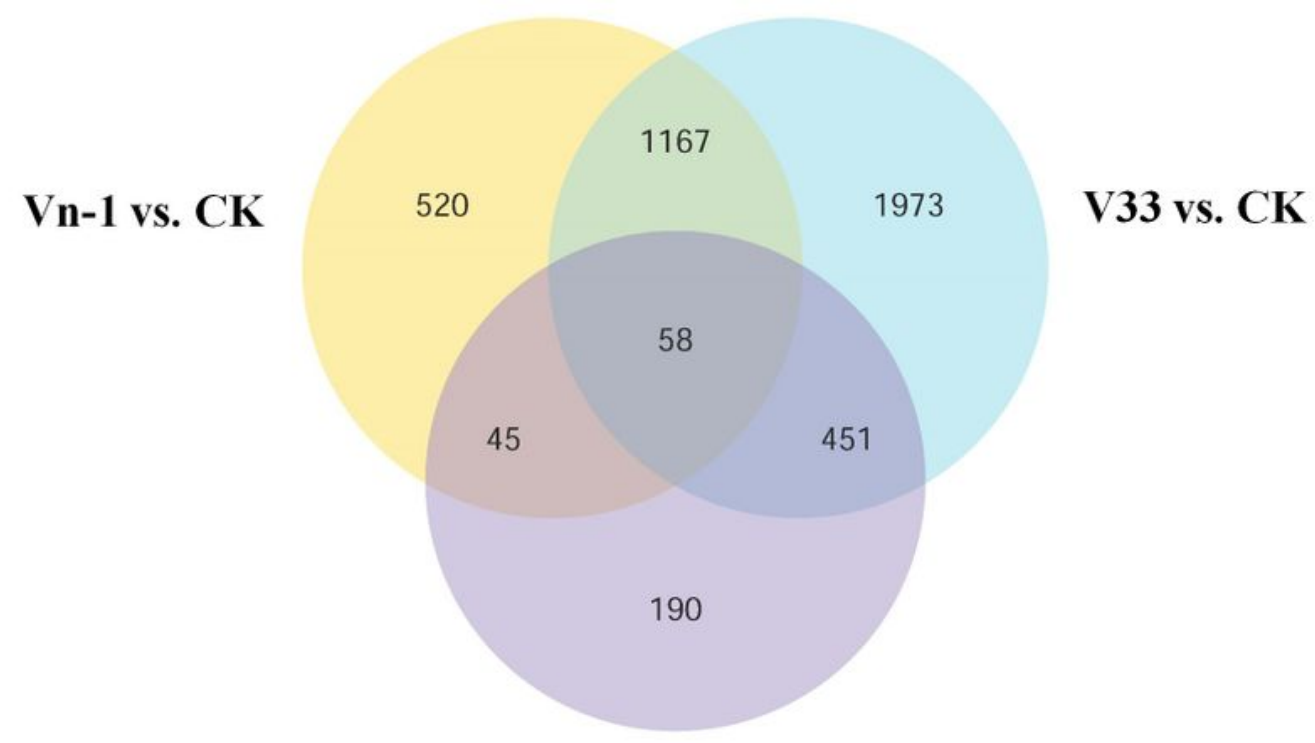

Vn-1 vs. V33

\section{Figure 4}

Differentially expressed genes (DEGs) in the three treatment groups A. Volcano plot of DEGs. The x-axis indicates gene expression fold changes between different strains, and the $y$-axis indicates the significance level of gene expression differences based on Benjamini-Hochberg-adjusted P-values. Significantly up- and down-regulated genes are indicated by red and green, respectively. B. Venn diagram of DEGs. The sum of the numbers in each circle is the total number of expressed genes within a 
comparison (Vn-1 vs. CK, V33 vs. CK, or Vn-1 vs. V33), whereas the number in an overlapping region is the number of expressed genes shared among indicated comparison groups.

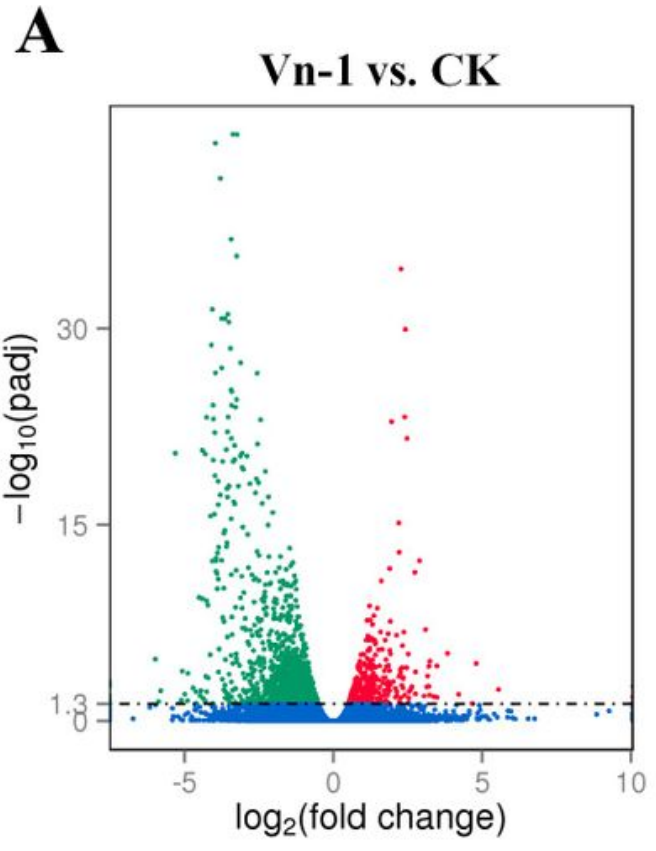

Differential Expressed Genes ( 1790 )

up regulated: 404

down regulated: 1386

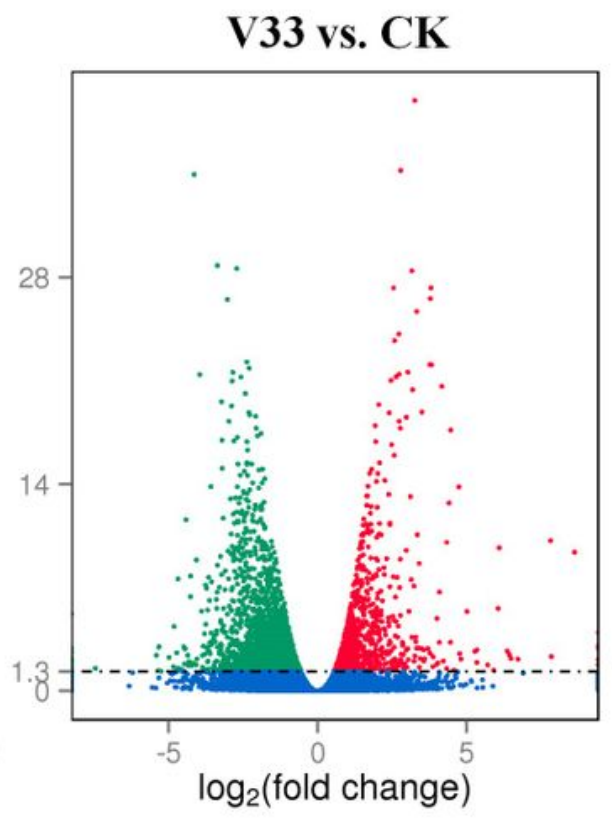

Differential Expressed Genes ( 3649 )

up regulated: 1204

down regulated: 2445

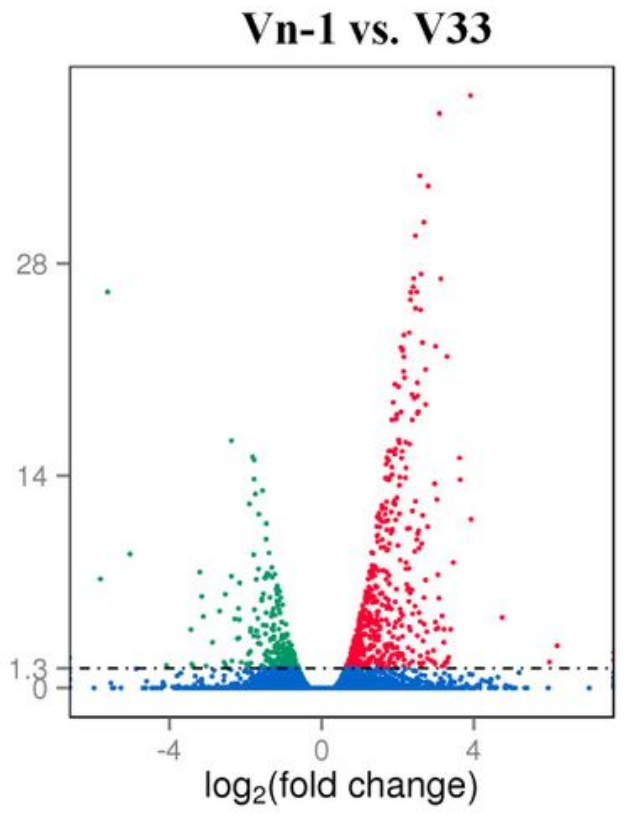

Difterential Expressed Genes ( 744 )

up regulated: 501

down regulated: 243

B

1167

520

1973

\section{V33 vs. CK}

Vn-1 vs. CK

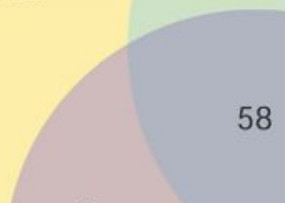

45
451

\section{Vn-1 vs. V33}

\section{Figure 4}

Differentially expressed genes (DEGs) in the three treatment groups A. Volcano plot of DEGs. The x-axis indicates gene expression fold changes between different strains, and the $y$-axis indicates the significance level of gene expression differences based on Benjamini-Hochberg-adjusted P-values. 
Significantly up- and down-regulated genes are indicated by red and green, respectively. B. Venn diagram of DEGs. The sum of the numbers in each circle is the total number of expressed genes within a comparison (Vn-1 vs. CK, V33 vs. CK, or Vn-1 vs. V33), whereas the number in an overlapping region is the number of expressed genes shared among indicated comparison groups.
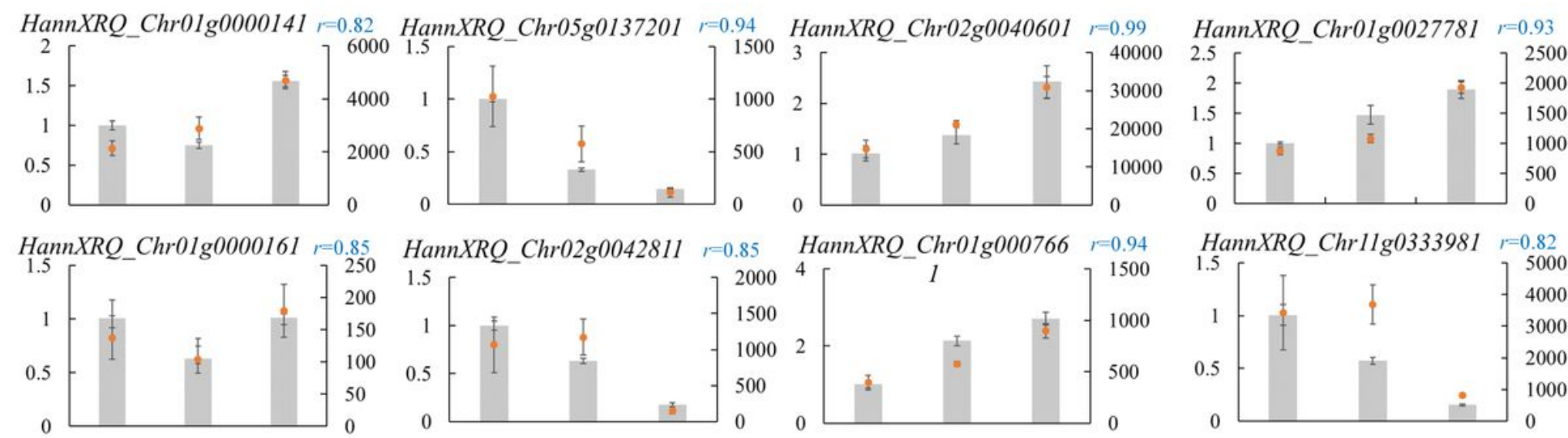

HannXRQ_Chrl6g0517151 $r=0.96$
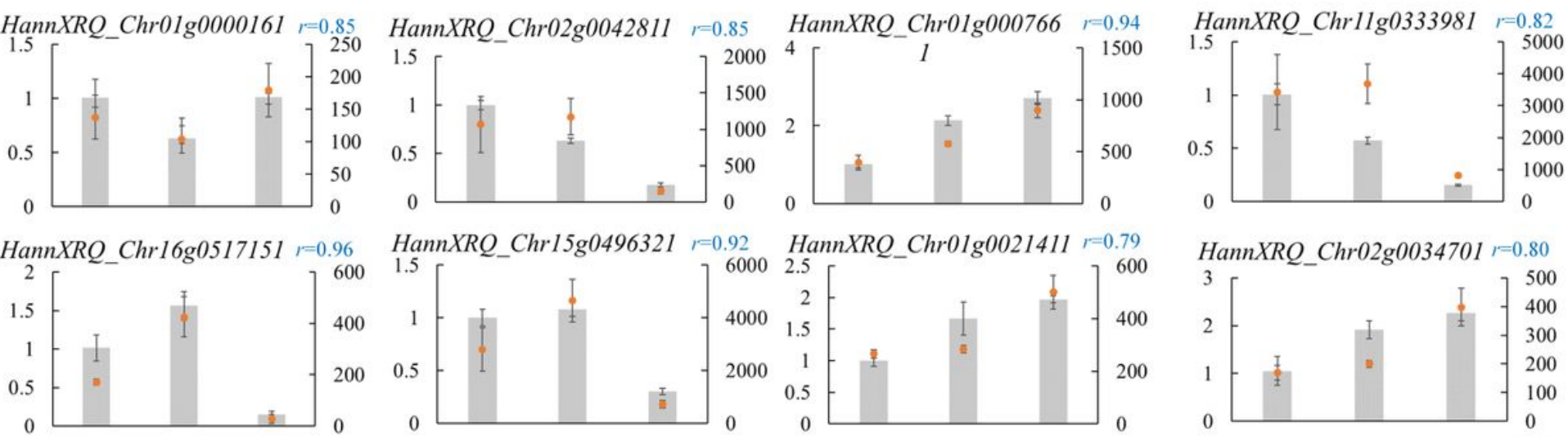

HannXRQ_Chr01g0019551 $r=0.92$
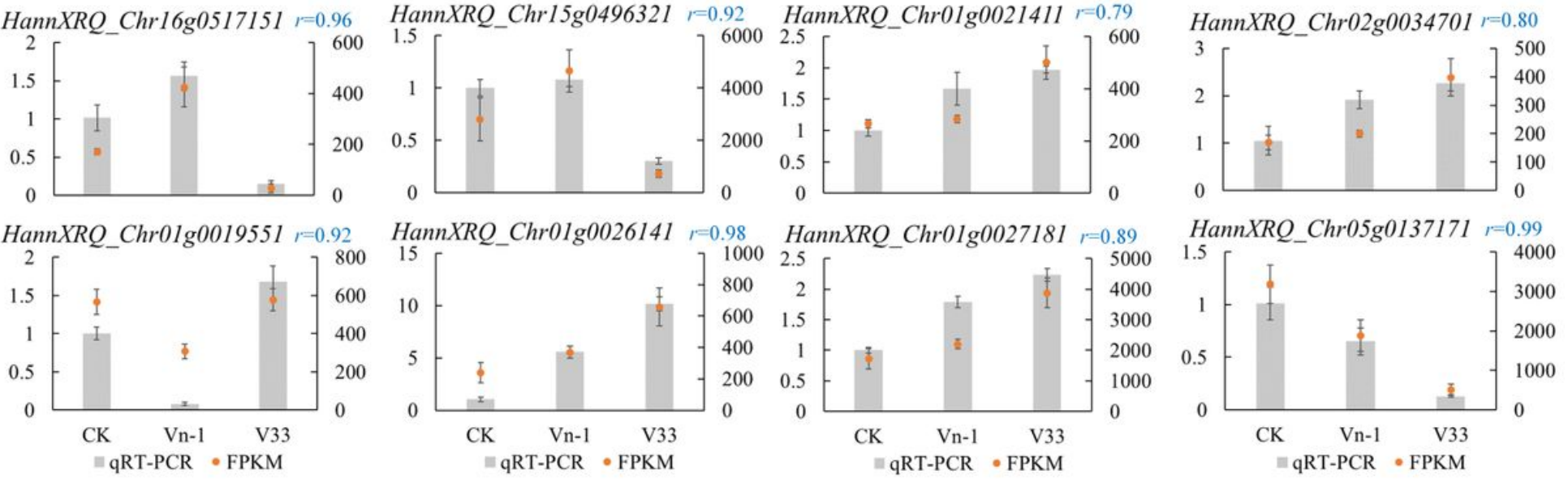

\section{Figure 5}

Validation of DEGs by qRT-PCR In each plot, expression levels of a given gene in CK, $\mathrm{Vn}-1$, and V33 samples are shown. Left and right y-axes indicate relative gene expression levels based on qRT-PCR analysis and FPKM values based on RNA-seq data, respectively. Columns and points correspond to mean values of the qRT-PCR and FPKM data, respectively, and r values are Pearson's correlation coefficients between data groups. Error bars represent standard deviations based on three replicates. 


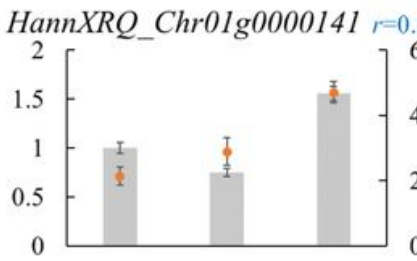

HannXRQ_Chr01g0000161 $r=0.85$

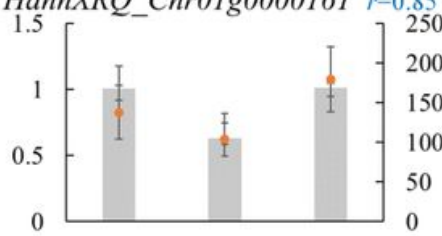

HannXRQ_Chr16g0517151 r =0.96

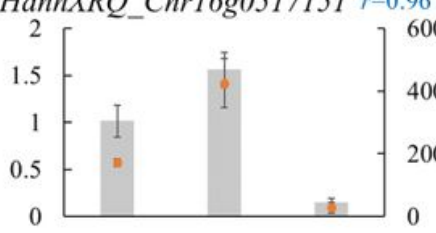

HannXRQ Chr01g0019551 $r=0.92$
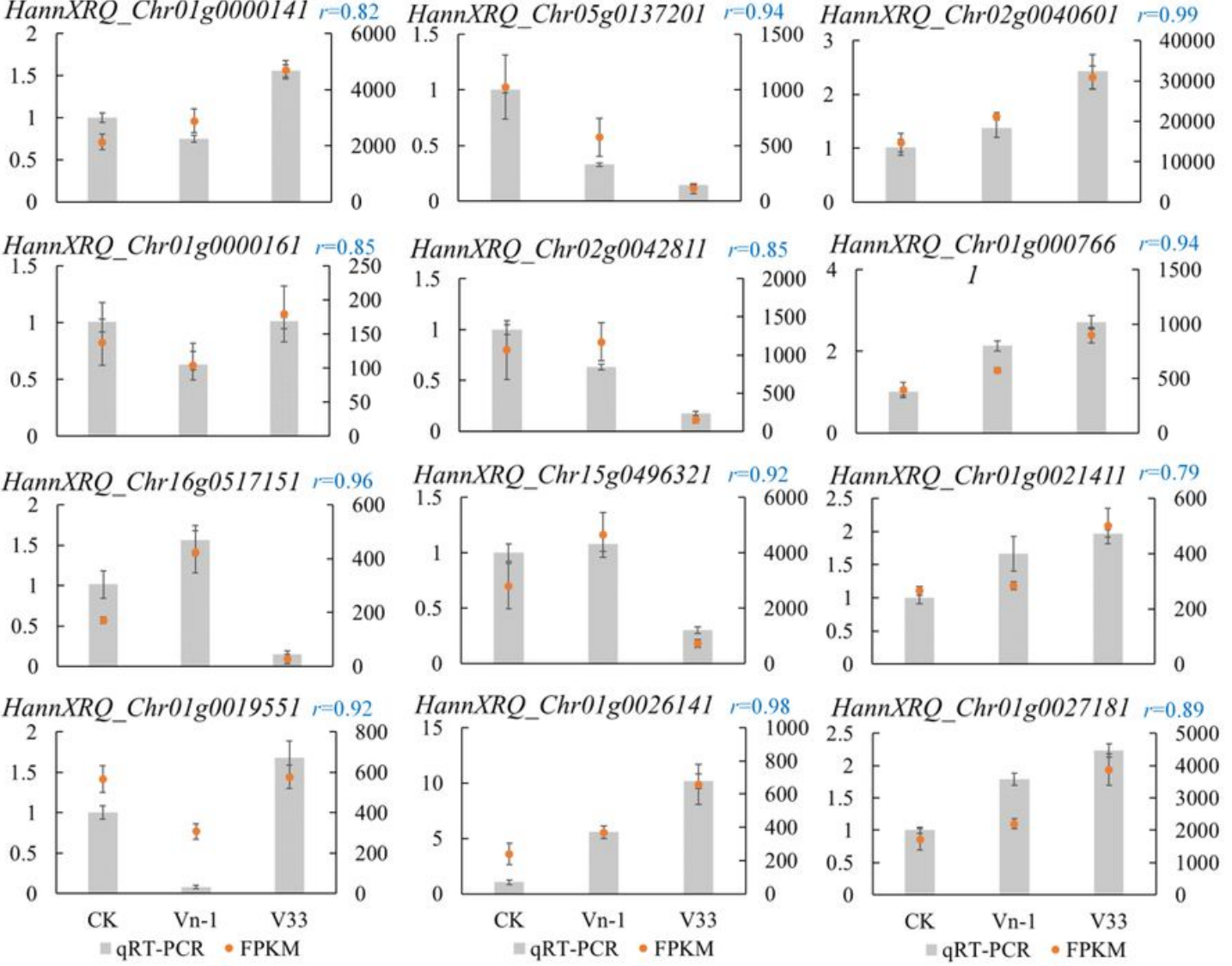
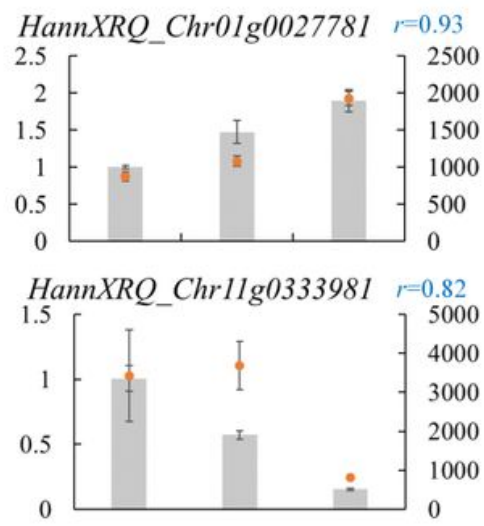

HannXRQ Chr02g0034701 $r=0.80$
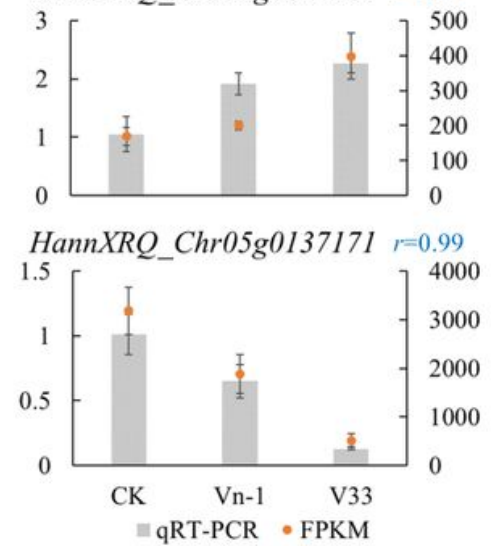

Figure 5

Validation of DEGs by qRT-PCR In each plot, expression levels of a given gene in CK, Vn-1, and V33 samples are shown. Left and right y-axes indicate relative gene expression levels based on qRT-PCR analysis and FPKM values based on RNA-seq data, respectively. Columns and points correspond to mean values of the qRT-PCR and FPKM data, respectively, and $r$ values are Pearson's correlation coefficients between data groups. Error bars represent standard deviations based on three replicates. 

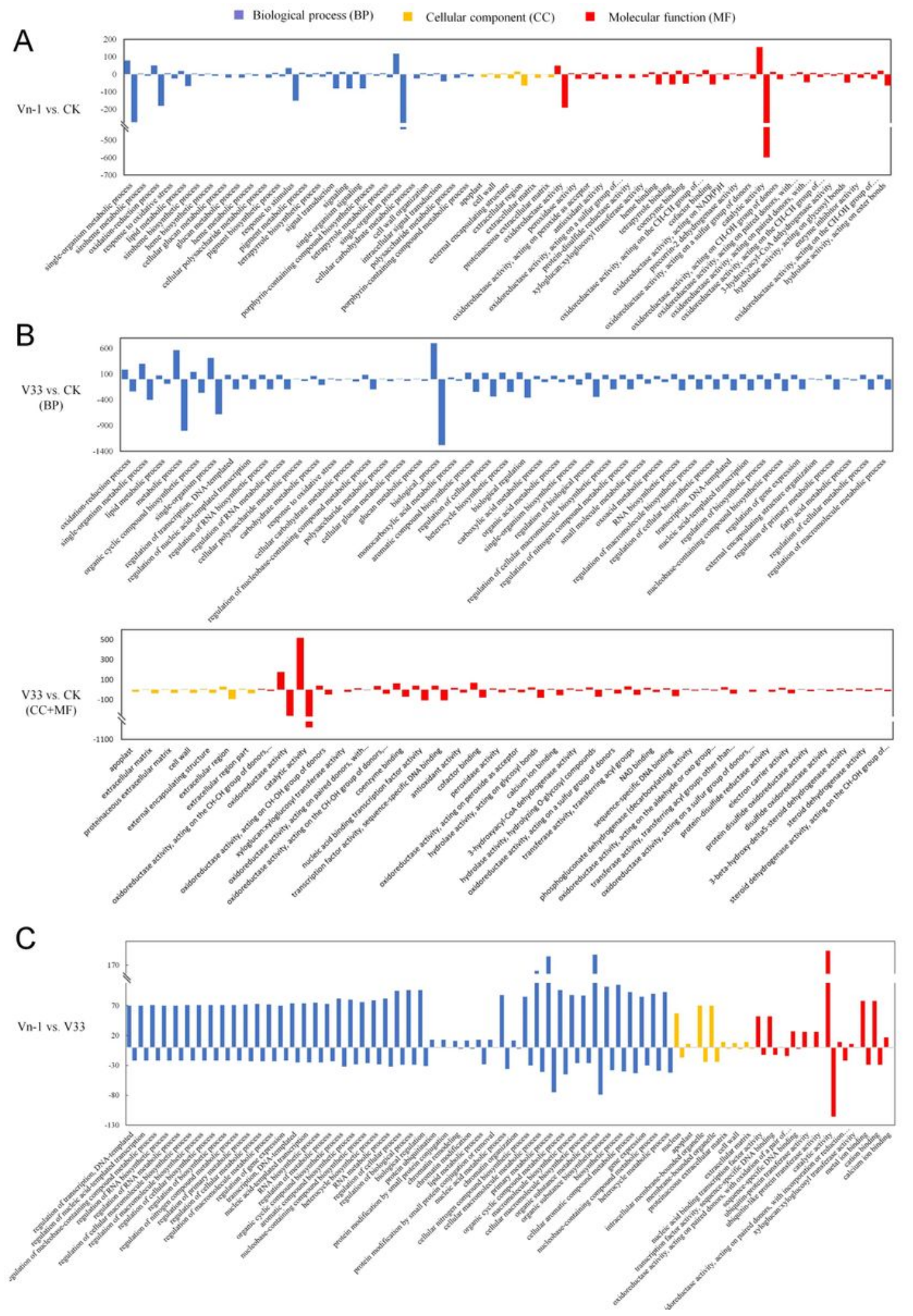

\section{Figure 6}

GO enrichment analysis of DEGs GO terms enriched in DEGs in Vn-1 vs. CK (A), V33 vs. CK (B), and Vn-1 vs. V33 (C) comparison groups. Biological process (blue), cellular component (yellow), and molecular function (red) categories are shown. The y-axis corresponds to the number of upregulated (+) and downregulated (-) DEGs. 

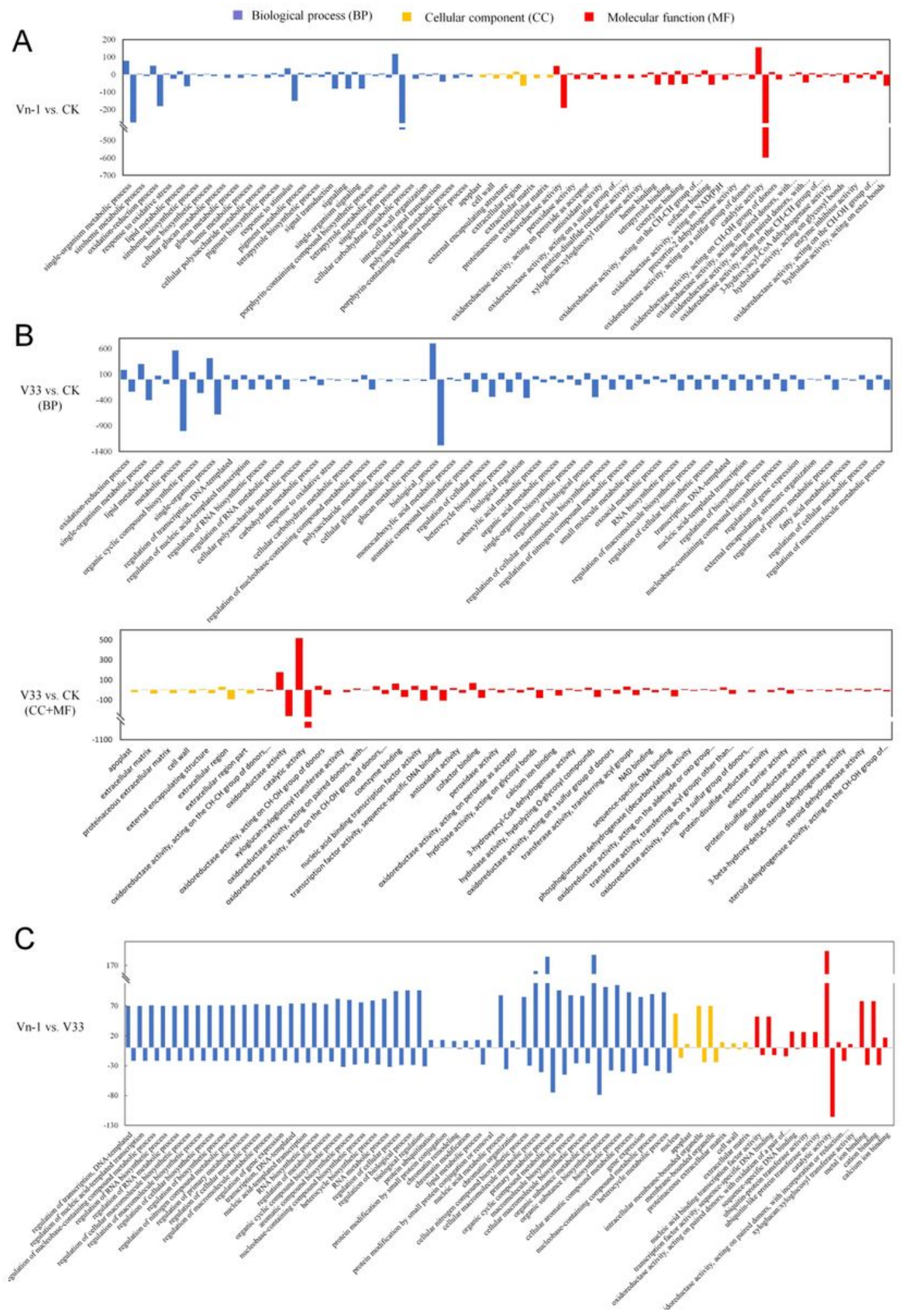

\section{Figure 6}

GO enrichment analysis of DEGs GO terms enriched in DEGs in Vn-1 vs. CK (A), V33 vs. CK (B), and Vn-1 vs. V33 (C) comparison groups. Biological process (blue), cellular component (yellow), and molecular function (red) categories are shown. The y-axis corresponds to the number of upregulated (+) and downregulated (-) DEGs. 
GO:0019354,GO:0046156, GO:0006783,GO:0042168, GO:0046148,GO:0050896, GO:0033014,GO:0007165, GO:0023052,GO:0044700, GO:0006779,GO:0033013, GO:0035556,GO:0006778, GO:0020037,GO:0046906, GO:0016651,GO:0043115, GO:0016705,GO:0016627, GO:0042440,GO:0004857, GO:0016788
GO:0008152,GO:1901362,GO:0006355,GO:1903506,GO:2001141, GO:0051252,GO:0005975,GO:0019219,GO:0008150,GO:0032787, GO:0019438,GO:0050794,GO:0018130,GO:0065007,GO:0019752, GO:0006082,GO:0044711,GO:0050789,GO:2000112,GO:0051171, GO:0044281,GO:0043436,GO:0032774,GO:0010556,GO:0031326, GO:0006351,GO:0097659,GO:0009889,GO:0034654,GO:0010468, GO:0045229,GO:0080090,GO:0006631,GO:0031323,GO:0060255, GO:0019222,GO:0008610,GO:0009664,GO:0071669,GO:0016070, GO:0044421.GO:0001071,GO:0003700,GO:0005509,GO:0004553, GO:0016746,GO:0051287,GO:0043565,GO:0004616,GO:0016620, GO:0016747,GO:0009055,GO:0015035,GO:0015036,GO:0003854, GO:0016229,GO:0033764

GO:0055114,GO:0006979,GO:0044710,GO:0006629,GO:0006073,GO:0044042,GO:0044264,GO:0044699,GO:0044262,GO:0071555,GO:0005976, GO:0048046,GO:0005618,GO:0030312,GO:0005576,GO:0031012,GO:0005578,GO:0016491,GO:0004601,GO:0016684,GO:0016209,GO:0016668, GO:0047134,GO:0016762,GO:0050662,GO:0016628,GO:0048037,GO:0016667,GO:0003824,GO:0016614,GO:0016717,GO:0003857,GO:0016798, GO:0016616

\section{Figure 7}

Enriched GO terms in Vn-1 vs. CK and V33 vs. CK comparison groups A total of $34 \mathrm{GO}$ terms were enriched in both comparison groups. GO terms belonging to biological process, cellular component, and molecular function categories are indicated in blue, yellow, and red, respectively. The number in each circle is the number of enriched $\mathrm{GO}$ terms in the indicated comparison group, and the number in the overlapping region is the number of $\mathrm{GO}$ terms enriched in both Vn-1 vs. CK and V33 vs. CK comparison groups.

\begin{tabular}{|c|c|c|c|}
\hline $\begin{array}{l}\text { GO: } 0019354, \mathrm{GO}: 0046156, \\
\text { GO: } 0006783, \mathrm{GO}: 0042168, \\
\text { GO: } 0046148, \mathrm{GO}: 0050896, \\
\text { GO:0033014,GO:0007165, } \\
\text { GO:0023052,GO:0044700, } \\
\text { GO:0006779, GO:0033013, } \\
\text { GO:0035556,GO:0006778, } \\
\text { GO:0020037,GO:0046906, } \\
\text { GO:0016651,GO:0043115, } \\
\text { GO:0016705,GO:0016627, } \\
\text { GO:0042440,GO:0004857, } \\
\text { GO:0016788 }\end{array}$ & Vn-1 vs. CK & V33 vs. CK & \begin{tabular}{|} 
GO:0008152,GO:1901362,GO:0006355,GO:1903506,GO:2001141, \\
GO:0051252,GO:0005975,GO:0019219,GO:0008150,GO:0032787, \\
GO:0019438,GO:0050794,GO:0018130,GO:0065007,GO:0019752, \\
GO:0006082,GO:0044711,GO:0050789,GO:2000112,GO:0051171, \\
GO:0044281,GO:0043436,GO:0032774,GO:0010556,GO:0031326, \\
GO:0006351,GO:0097659,GO:0009889,GO:0034654,GO:0010468, \\
GO:0045229,GO:0080090,GO:0006631,GO:0031323,GO:0060255, \\
GO:0019222,GO:0008610,GO:0009664,GO:0071669,GO:0016070, \\
GO:0044421,GO:0001071,GO:0003700,GO:0005509,GO:0004553, \\
GO:0016746,GO:0051287,GO:0043565,GO:0004616,GO:0016620, \\
GO:0016747,GO:0009055,GO:0015035,GO:0015036,GO:0003854, \\
GO:0016229,GO:0033764
\end{tabular} \\
\hline
\end{tabular}

GO:0055114,GO:0006979,GO:0044710,GO:0006629,GO:0006073,GO:0044042,GO:0044264,GO:0044699,GO:0044262,GO:0071555,GO:0005976, $\mathrm{GO}: 0048046, \mathrm{GO}: 0005618, \mathrm{GO}: 0030312, \mathrm{GO}: 0005576, \mathrm{GO}: 0031012, \mathrm{GO}: 0005578, \mathrm{GO}: 0016491, \mathrm{GO}: 0004601, \mathrm{GO}: 0016684, \mathrm{GO}: 0016209, \mathrm{GO}: 0016668$, GO:0047134,GO:0016762,GO:0050662,GO:0016628,GO:0048037,GO:0016667,GO:0003824,GO:0016614,GO:0016717,GO:0003857,GO:0016798, GO:0016616

\section{Figure 7}

Enriched GO terms in Vn-1 vs. CK and V33 vs. CK comparison groups A total of $34 \mathrm{GO}$ terms were enriched in both comparison groups. GO terms belonging to biological process, cellular component, and molecular function categories are indicated in blue, yellow, and red, respectively. The number in each circle is the number of enriched GO terms in the indicated comparison group, and the number in the overlapping region is the number of $\mathrm{GO}$ terms enriched in both $\mathrm{Vn}-1$ vs. CK and V33 vs. CK comparison groups. 


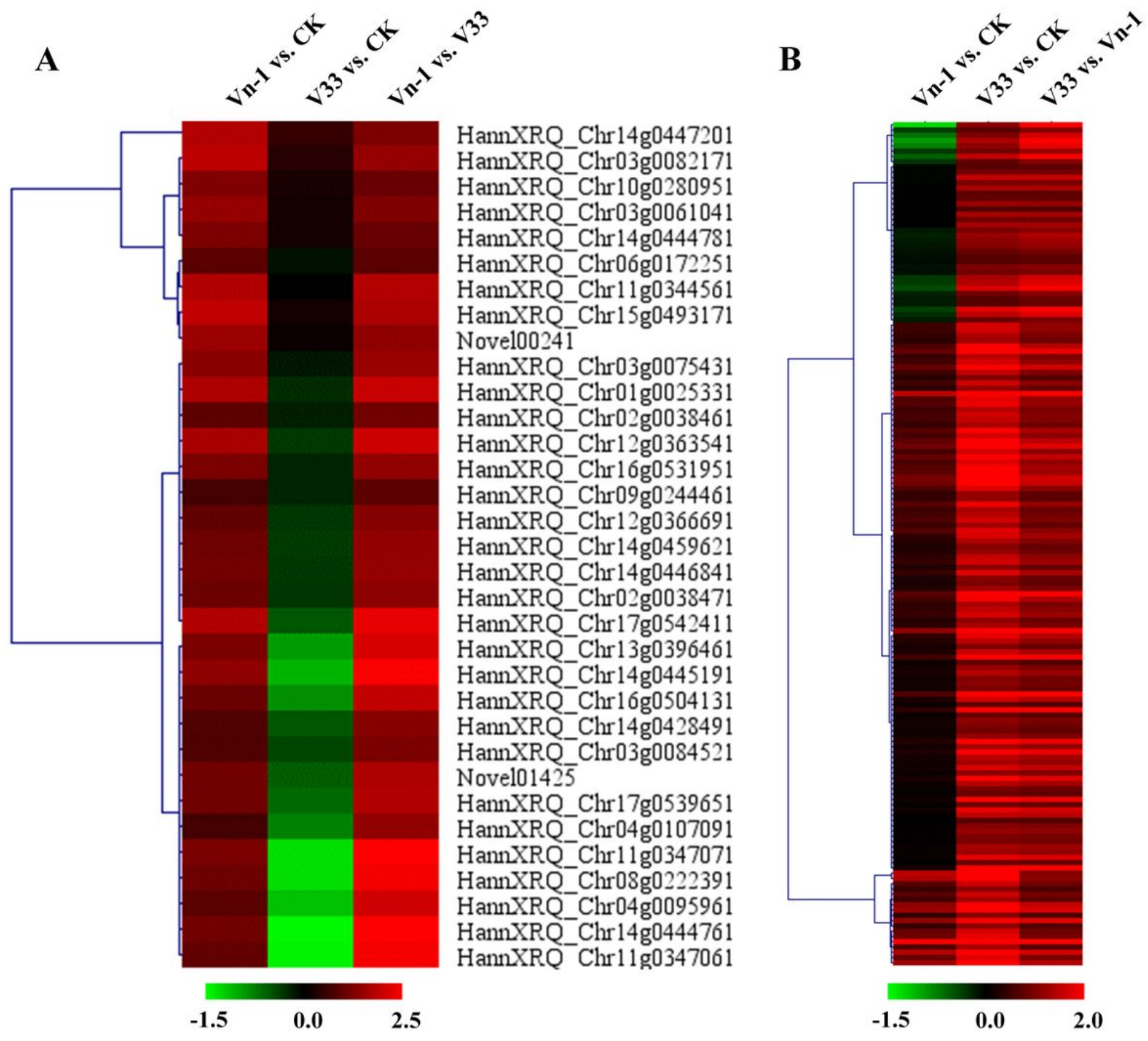

\section{Figure 8}

Heat map of predicted Verticillium wilt resistance and susceptibility genes in sunflower Heat maps of 33 predicted resistance genes (B) and 160 predicted susceptibility genes (B) identified by comparative transcriptome analysis. Genes upregulated in Vn-1 vs. CK and Vn-1 vs. V33 comparison groups but downregulated or not significantly changed in the V33 vs. CK group were predicted to be resistance genes. Genes upregulated in V33 vs. CK and V33 vs. Vn-1 groups but downregulated or unchanged in the Vn-1 vs. CK group were predicted to be susceptibility genes. 


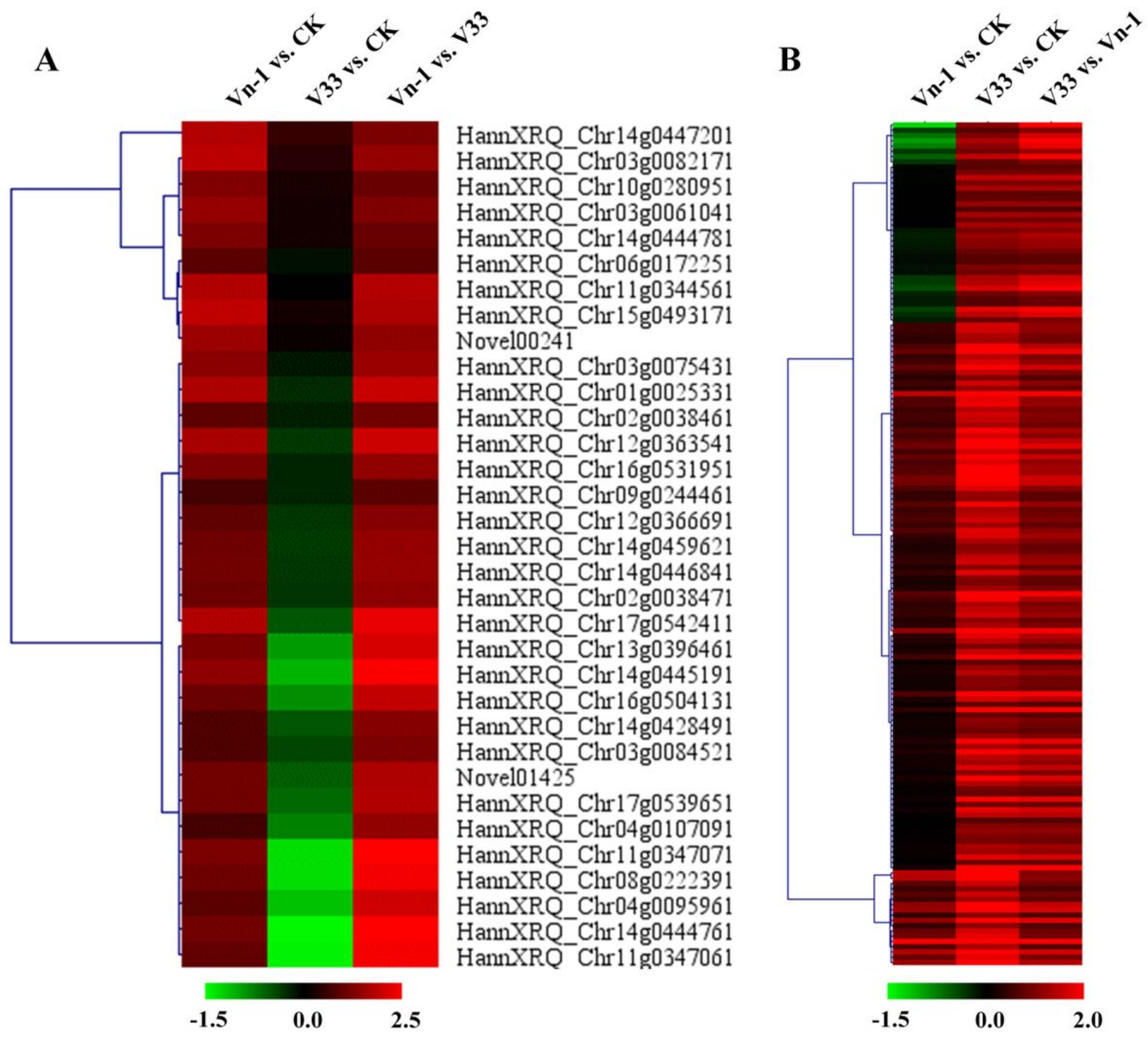

\section{Figure 8}

Heat map of predicted Verticillium wilt resistance and susceptibility genes in sunflower Heat maps of 33 predicted resistance genes (B) and 160 predicted susceptibility genes (B) identified by comparative transcriptome analysis. Genes upregulated in Vn-1 vs. CK and Vn-1 vs. V33 comparison groups but downregulated or not significantly changed in the V33 vs. CK group were predicted to be resistance genes. Genes upregulated in V33 vs. CK and V33 vs. Vn-1 groups but downregulated or unchanged in the Vn-1 vs. CK group were predicted to be susceptibility genes.

\section{Supplementary Files}


This is a list of supplementary files associated with this preprint. Click to download.

- FigS1.docx

- FigS1.docx

- Figs2.docx

- Figs2.docx

- Figs3.docx

- FigS3.docx

- FigS4.docx

- Figs4.docx

- SuplementaryTalbes.xls

- SuplementaryTalbes.xls 